\title{
Stellar envelopes as sources of broad line region emission: New possibilities allowed
}

\author{
G. Torricelli-Ciamponi ${ }^{1}$ and P. Pietrini ${ }^{2}$ \\ 1 Osservatorio Astrofisico di Arcetri, Largo E. Fermi 5, 50125 Firenze, Italy \\ 2 Dipartimento di Astronomia e Scienza dello Spazio, Università di Firenze, Largo E. Fermi 2, 50125 Firenze, Italy
}

Received 19 February 2001 / Accepted 5 August 2002

\begin{abstract}
In Active Galactic Nuclei (AGNs) the presence of a star cluster around the central black hole can have several effects on the dynamics and the emission of the global system. In this paper we analyze the interaction of stellar atmospheres with a wind outflowing from the central region of the AGN nucleus. Even a small mass loss from stars, as well as possible star collisions, can give a non-negligible contribution in feeding matter into the AGN nuclear wind. Moreover, stellar mass loss can produce envelopes surrounding stars that turn out to be suitable for reproducing the observed emission from the Broad Line Region (BLR). In this framework, the envelope can be confined by the bow shock arising from the interaction between the expanding stellar atmosphere and the AGN nuclear wind.
\end{abstract}

Key words. radiation mechanisms: thermal - ultraviolet: galaxies - galaxies: nuclei - galaxies: seyfert - quasars: general

\section{Introduction}

It is generally accepted that the broad emission lines present in AGN spectra originate in numerous, small and relatively cold gas concentrations in the neighborhood of the central black hole, the so-called Broad Line Region (BLR). The observed line properties directly indicate the existence of such gas concentrations; however, the physical nature of these structures is still a matter of debate. Up to the present day, three possible classes of models have been envisaged: gas clouds, accretion disks, and stellar wind envelopes. Each scenario suffers from still unclarified problems and we refer to the recent review by Korista (1999) for a presentation of the problem.

In general, broad emission line regions in AGNs turn out to be characterized by a relatively narrow range in ionization parameter $U$, i.e., $U \equiv L /\left[4 \pi c r^{2} n_{*}<h v>\right] \simeq 0.01-1$ (Alexander \& Netzer 1994; Peterson 1997; Netzer 1990). In this expression $r$ is the distance from the central black hole, $n_{*}$ is a representative value of the number density of the line emitting gas, $L$ is the ionizing radiation field luminosity from a central source, illuminating the line emitting region, and $\langle h v\rangle$ is the average photon energy of the ionizing radiation field. A first estimate of the conditions of the line emitting gas, such as $n_{*}$ and $U$, can be obtained from an analysis of the emission spectrum; coupling this information with the evaluation of the luminosity and of the ionizing spectrum, the relation above, defining $U$, can be inverted to give an estimate of the characteristic size of the $\mathrm{BLR}$, or, better, the characteristic distance of the line emitting

Send offprint requests to: $\mathrm{P}$. Pietrini, e-mail: paola@arcetri.astro.it gas from the central continuum source, $r_{\mathrm{BLR}}$ (see Wandel et al. 1999). In a first, simplistic use of photoionization arguments, under the assumption that the ionizing continuum shape is quite similar and both $n_{*}$ and $U$ are substantially the same for all the AGNs (see Kaspi et al. 2000), a relationship, between the characteristic BLR size and the AGN luminosity, of the type $r_{\mathrm{BLR}} \propto L^{0.5}$ would then be expected. Indeed, this would just be an order of magnitude evaluation, since there are reliable indications that the BLR material density and the ionization parameter are not constant at all even across the BLR extension of a single AGN (see, e.g., Kaspi et al. 2000). Nonetheless, this was actually what reverberation mapping studies of several AGNs seemed to suggest until very recently, implying $r_{\mathrm{BLR}} \simeq$ $0.1 L_{46}^{1 / 2}$ pc (Netzer \& Peterson 1997; Kaspi 1997) (where $L_{46}$ is the AGN luminosity in units of $10^{46} \mathrm{erg} / \mathrm{s}$ ). In a reverberation technique study of a sample of 17 quasars, combining their data with those available for Seyfert 1 galaxies, Kaspi et al. (2000) propose a significantly different relationship between $r_{\mathrm{BLR}}$ and the luminosity of the AGN, namely $r_{\mathrm{BLR}} \propto L^{0.7}$. Although the exponent does not differ much from the previous one, it is remarkable that the ensemble of the data fit by the latter relation, are not fit by the one with an 0.5 exponent.

In a previous paper (Pietrini \& Torricelli 2000, hereafter Paper I), motivated by recent observations supporting radial outflows even in radio-quiet AGN's, such as Seyfert 1s, (Crenshaw et al. 1999; Weymann et al. 1997), we have analysed the physical structure and characteristics of a global AGN outflow, presumably originating in the very central regions and expanding out to large distances as a kind of background/connection for various observational components of the 
AGN structure [BLR, UV absorbers, X-ray “warm absorbers"]. A solution for the wind equations, accounting for the physical requirements typical of central regions of Seyfert-like AGN, turned out to be possible only under rather specific conditions. One of the resulting constraints is that the outflowing wind may exist only if its density is rather low. The only possibility to increase the wind density is to feed matter into the wind itself in a certain range of distances from the nucleus (see Sect. 8.4 of Paper I for details).

As far as the BLR cloud model is concerned, the presence of a nuclear wind generally augments problems of cloud survival, rather than solving them, unless the clouds are somehow comoving with the outflow itself. In fact, one problem for the cloud existence is the drag force at work between the moving clouds and a non-comoving confining medium. This drag force would rapidly disrupt the clouds. The observationally inferred information on the cloud kinematics indicates that this motion is not radially directed and it must be characterized by a more complex velocity field. Also, the possibility of a Keplerian pattern has been suggested by Wandel et al. (1999). Since, for the reasons explained above, our choice is to work within the scenario of a radially directed nuclear outflow, the cloud survival problem would inevitably be worsened by the presence of a radial nuclear wind.

For the stellar wind envelopes, since they are continuously fed by the stellar wind, disruption is not a problem; another velocity component in their relative motion with respect to the interstellar medium does not constitute a problem either. On the contrary, we will show in this paper that this new component in the relative motion of the envelopes with respect to the surrounding medium can be a fundamental factor; in fact, it can contribute significantly to confine the plasma envelopes via the formation of bow-shock fronts.

The above considerations, together with the necessity of feeding matter in the AGN wind, induce us to invoke stellar envelopes as a possible interpretation of the nature of BLR emitting plasma. In addition, the virial assumption of Keplerian motions used to interpret the emission line width (Wandel et al. 1999 ) is in good agreement with the stellar origin of BLR emission. Indeed, this possibility has been widely discussed in the literature. In particular, after two pioneering works of Scoville \& Norman (1988) and Kazanas (1989), the star model has been reanalyzed by Alexander \& Netzer (1994, hereafter AN1), and Alexander \& Netzer (1997, hereafter AN2), who introduce "bloated" stars as stars characterized by a very extended envelope, but, due to the specific AGN environment, different from supergiant stars as known in the solar neighbourhood. We refer to AN1 for a wider presentation of the scenario and its problems.

In their papers, Alexander \& Netzer (AN1, AN2) present different possibilities for confining "bloated" star envelopes and conclude that the more effective one is tidal disruption by the black hole. However, AN1 and AN2 assume in their works that the stellar winds expand into vacuum, asserting that this does not constitute a limitation on the validity of the analysis. On the contrary, in this paper we analyze whether and how the presence of a nuclear AGN wind, such as the one described in Paper I, can have an influence on the BLR physical structure.
In particular, by inserting mass losing stars in their specific environment and investigating the interaction of the stellar wind with the AGN wind, we introduce another confining mechanism for the star envelopes. We then compare it to both the tidal one and to the other possible confinement mechanisms investigated by AN1 and AN2. Finally we analyze the consequences for BLR parameter values.

In Sect. 2, we discuss confinement of the expanding stellar envelopes and introduce the bow-shock formation mechanism as a further means of defining an outer boundary for these envelopes. Section 3 is devoted to the connection and interaction between the AGN nuclear wind, as developed in Paper I, and the central star cluster, with colliding and mass losing stars, in terms of the resulting mass deposition into the AGN nuclear wind. In Sect. 4, we describe the physical parameters that turn out to be involved in the computation of the model, their significance in the outcome determination and the general properties of a stellar envelope necessary to produce the observed line emission. We then identify a sort of "observational test" for the results of a BLR model, through the comparison with typical values inferred from observations of global BLR parameters, such as the covering factor, the characteristic BLR radius, the ionization parameter, and the fact that no broad forbidden lines are observed. Section 5 discusses how the identified general requirements put physical limitations on our model parameters. The general results of our model are presented in Sects. 5.15.3 for three distinct cases, that differ basically in the choice of the mass loss rates for the various types of stars in the central cluster. Section 6 is devoted to general discussion of the main results, including a comparison with the works of other authors applying different models to infer the general properties of the BLR of a specific source, NGC 5548, which is well representative of the Seyfert 1 class of AGNs. Finally, in Sect. 7 we summarize our results and the general features that characterize our interpretation of the BLR, highlighting the qualities and the limitations of the model.

\section{Confinement of the stellar envelopes: Bow shocks around moving stars versus other mechanisms}

When a mass-losing star moves rapidly through the interstellar medium, its wind interacts with the surrounding plasma, giving rise to an elongated bow shock. The physics of these shock fronts has been widely studied in different contexts (see e.g. Perry \& Dyson 1985; van Buren et al. 1990 and references therein) for different purposes. In general, we expect that the expanding stellar wind can develop a shock front, in which the wind kinetic energy is partially converted and radiated away. Therefore, in a stationary configuration, the shock position is determined by the equilibrium of the external pressure and that of the stellar wind. The resulting shock geometry is a cometarylike tail elongated in the direction opposite to that of the star motion.

In our case the situation is more complex since stars move around the AGN nucleus with a velocity $\boldsymbol{V}(r)$ and are embedded in a radial nuclear wind characterized by a number density $n_{\mathrm{w}}(r)$ and expanding with velocity $\boldsymbol{v}_{\mathrm{w}}(r)=v_{\mathrm{w}}(r) \hat{\boldsymbol{e}}_{\mathrm{r}}$. 
The AGN wind velocity $v_{\mathrm{w}}(r)$, number density $n_{\mathrm{w}}(r)$ and temperature $T_{\mathrm{w}}(r)$ profiles are those computed through the resolution of the system of stationary hydrodynamical equations, described in Paper I, including the appropriate mass deposition. The nuclear wind velocity $v_{\mathrm{w}}$ undergoes the transition from sub-sonic to super-sonic at a distance $r_{\mathrm{c}}$ from the AGN nucleus, where $r_{\mathrm{c}} / r_{\mathrm{g}} \geq 1 / 4\left(c / c_{\mathrm{s}}\right)^{2}$, with $c_{\mathrm{s}}$ being the local sound speed, $c$ the light speed, and $r_{\mathrm{g}} \equiv 2 G M_{\mathrm{BH}} / c^{2}$ the black hole Schwarschild radius.

For the stellar velocity we adopt a Keplerian motion (consistent with recent interpretations of emission line width Wandel et al. 1999):

$V(r)=\sqrt{\frac{G M(r)}{r}}$,

where $M(r)$ is the total mass (central black hole plus star cluster) within the radius $r$, assuming its distribution is spherically symmetric. The velocity of the stars is generally supersonic inside the nuclear wind sonic radius, while, of course, $v_{\mathrm{w}}$ is supersonic outside. Hence, in our case the bow shock will have a different geometry depending on the regime in which it is computed: cometary-like shocks with the tail in the direction opposite to the star motion (when the ram pressure contribution is induced by the star motion, $m_{\mathrm{H}} n_{\mathrm{w}} V^{2}$ ); or with the tail extending radially outwards from the central black hole (when the ram pressure contribution is due to the AGN wind: $m_{\mathrm{H}} n_{\mathrm{w}} v_{\mathrm{w}}^{2}$ ); or a combination of the two. It is beyond the scope of this paper to investigate the detailed physics and geometry of this configuration; instead, what is important in this context is the existence of a region around the star where the stellar wind can be confined by this mechanism. For the sake of simplicity we have chosen to represent this complex wind envelope region schematically as a quasi-spherical structure, so that its geometrical extension can be characterized simply by a radius defining the radial distance of the envelope boundary from the star.

In order to derive this envelope radius, first we have to parameterize the physical properties of the expanding envelope, namely give a prescription for the density of the gas in the envelope itself and for its expansion velocity. Our simplifying choice is to adopt a radial power law behaviour for the stellar atmosphere expansion velocity:

$v_{*}(R)=v_{0}\left(\frac{R}{R_{*}}\right)^{\alpha} \quad$ for $R>R_{*}$,

where $R$, the distance from the star center, and $R_{*}$, the radius of the star, are both expressed in the same length units; this relation is taken as independent of the distance, $r$, of the star from the central black hole. Taking into account continuity equation for the envelope gas, we can derive its density as a function of $R$ as well:

$n_{\text {env }}(R)=\frac{\dot{M}_{*}}{4 \pi m_{\mathrm{H}} R^{2} v_{*}(R)}=\left(\frac{\dot{M}_{*}}{4 \pi m_{\mathrm{H}} v_{0} R_{*}^{2}}\right)\left(\frac{R}{R_{*}}\right)^{-(\alpha+2)}$.

Once these quantities are defined, the order of magnitude of the distance from the star, $R_{\text {bow }}$, at which the bow shock can be formed, can be determined by using the standard methods, that is from the two equations describing the stellar mass loss rate (i.e., continuity equation again) and the balance between the total pressure (thermal+ram pressure) of the stellar wind and that of the ambient medium, all evaluated at $R_{\text {bow }}$ :

$$
\begin{aligned}
& \dot{M}_{*}=4 \pi m_{\mathrm{H}} R_{\mathrm{bow}}^{2} n_{\mathrm{env}}\left(R_{\mathrm{bow}}\right) v_{*}\left(R_{\mathrm{bow}}\right) \\
& P_{*}+m_{\mathrm{H}} n_{\mathrm{env}}\left(R_{\mathrm{bow}}\right) v_{*}^{2}\left(R_{\mathrm{bow}}\right)=P_{\mathrm{w}}+P_{\mathrm{ram}} .
\end{aligned}
$$

Here we assume that $P_{*}=n_{\mathrm{env}} k T_{*} / \mu_{*}$ and $P_{\mathrm{w}}=n_{\mathrm{w}} k T_{\mathrm{w}} / \mu_{\mathrm{w}}$ are the thermal pressures of the star and of the AGN winds, respectively, where $n_{i} \equiv \rho_{i} / m_{\mathrm{H}}$ (for $i=$ env, w), with $\rho_{i}$ the mass density. We have taken $\mu_{*}=\mu_{\mathrm{w}}=0.6$, as expected for a fully ionized plasma with cosmic abundances (see Allen 1985); note that in the following we indicate both $\mu_{*}$ and $\mu_{\mathrm{w}}$ as $\mu$. Also, $v_{*}$ is the expansion velocity of the stellar atmosphere as given by Eq. (2) for $R=R_{\text {bow }}$. The ram pressure of the external medium can be identified with the maximum of that due to the star motion and that due to the AGN wind:

$P_{\mathrm{ram}}=\operatorname{Max}\left[m_{\mathrm{H}} n_{\mathrm{w}} V^{2}, m_{\mathrm{H}} n_{\mathrm{w}} v_{\mathrm{w}}^{2}\right]=m_{\mathrm{H}} n_{\mathrm{w}} v_{\mathrm{Max}}^{2}$.

The two equations above (Eqs. (4) and (5)) turn out to depend on the distance of the star (whose envelope we are describing) from the central black hole, $r$, since they contain physical quantities depending on $r$ itself. These are the ones defining the AGN nuclear wind $\left(n_{\mathrm{w}}(r), T_{\mathrm{w}}(r), v_{\mathrm{w}}(r)\right)$, and the Keplerian velocity of the star in the local gravitational field, as given by Eq. (1). Also, these quantities can be combined so as to define global envelope properties as functions of $r$ as well. In fact, Eq. (4) can be rewritten as

$n_{*}(r) \equiv n_{\mathrm{env}}\left(R_{\mathrm{bow}}\right)=\frac{\dot{M}_{*}}{4 \pi m_{\mathrm{H}} R_{\mathrm{bow}}^{2} v_{*}}$

and, making use of Eq. (6), Eq. (5) becomes

$R_{\mathrm{bow}}^{2}(r)=\frac{\dot{M}_{*}}{4 \pi m_{\mathrm{H}} n_{\mathrm{w}}(r) v_{*}} \frac{m_{\mathrm{H}} v_{*}^{2}+k T_{*} / \mu}{m_{\mathrm{H}} v_{\mathrm{Max}}^{2}(r)+k T_{\mathrm{w}} / \mu}$,

where $v_{*}$ indicates the value of the stellar atmosphere velocity at its boundary $R_{\text {bow }}$, as in Eq. (5). Note that in Eq. (6) we have redefined $n_{\text {env }}\left(R_{\text {bow }}\right)$, the density of the stellar envelope at its external boundary, as $n_{*}(r)$, explicitly showing its dependence on $r$, since its value does depend on the distance of the envelope from the black hole, as mentioned above. In the following, unless otherwise specified, we use $n_{*}(r)$ to indicate a reference number density value characterizing the stellar atmosphere. As for the stellar atmosphere gas temperature, here we assume for simplicity that it is constant, i.e. independent both of $r$ and of $R$; this assumption and its validity within the present framework are briefly discussed in Sect. 5. The two relationships shown above define the density and the extension of the stellar wind envelope as functions both of the stellar wind velocity and temperature, and of the external confining plasma parameters. Since the stellar wind velocity depends on $R_{\text {bow }}$, Eq. (7) is in general an implicit equation.

As Alexander \& Netzer (AN1, AN2) have shown, several other mechanisms in principle can be competing for the definition of the physical extension of the stellar wind envelope. In the following we briefly outline them, in order to compare 
the characteristic envelope radius values defined by each mechanism at any given radial distance $r$ from the black hole with $R_{\text {bow }}$, i.e. the distance from the star at which the bow shock, due to the interaction of the stellar wind with the nuclear AGN outflow, would form. The various physical processes that are to be examined produce a stellar wind envelope extension that is characterized by a different dependence on the radial distance $r$ from the central black hole. Therefore, for each value of $r$, we shall finally identify the effective extension of the envelope itself with the smallest radius obtained, thus selecting the most efficient mechanism for stellar wind confinement.

A first effect we need to take into account is the possibility of tidal disruption of the outer layers of the stellar envelope, due to the black hole gravitational pull. In this case the size of the stellar envelope is given by (see AN1)

$R_{\mathrm{tidal}} \simeq 2\left(\frac{M_{*}}{M_{\mathrm{BH}}}\right)^{1 / 3} r$

showing a simple linear dependence on the distance from the black hole.

Another possible definition of an upper limit to the stellar wind extension can be determined by the reasonable assumption of the existence of a finite mass in the stellar wind. Again following AN1, we suppose that the total mass in the stellar wind must not exceed $0.2 M_{\odot}$; this leads to the condition

$\int_{R_{*}}^{R_{\text {mass }}} \dot{M}_{*} \mathrm{~d} t=\int_{R_{*}}^{R_{\text {mass }}} \frac{\dot{M}_{*}}{v_{*}} \mathrm{~d} R=0.2 M_{\odot}$,

which, for a given stellar wind velocity field $v_{*}(R)$, defines $R_{\text {mass }}$ as the maximum stellar wind size allowed for, due to the limit on the stellar wind mass content. Assuming that Eq. (2) defines the velocity profile $v_{*}(R)$ for the stellar wind, we can explicitly solve the integral to obtain

$R_{\text {mass }}=\left[\left(\frac{(1-\alpha) 0.2 M_{\odot} v_{0}}{\dot{M}_{*}}+R_{*}\right) R_{*}^{-\alpha}\right]^{1 /(1-\alpha)} ;$

notice that in this specific case the stellar envelope extension would not depend on the radial distance $r$ from the central black hole.

Comptonization effects, due to the central ionizing continuum heating the outer layers of the stellar envelope, should also be taken into account in the determination of the external boundary of the envelope itself. This process (see Kazanas 1989, and AN1) induces complete ionization of the outer layers of the stellar wind, that become part of hot "Comptonized" phase. It is effective on the denser stellar wind down to layers in which the density of the envelope material reaches a critical value, $n_{\text {Comp }}$, above which, that is deeper into the envelope, a "cool phase" is still allowed (Kazanas 1989; Krolik et al. 1981), and therefore the stellar wind survives as such. This density value is defined by a critical value, $\Xi_{c}$, of the "ionization" parameter $\Xi \equiv L_{\text {ion }} /\left(4 \pi r^{2} c n k T\right)$ for the existence of a "cool" phase, for a given ionizing radiation field, and it is

$n_{\text {Comp }}(r)=\frac{1}{\Xi_{\mathrm{c}}} \frac{L_{\text {ion }}}{4 \pi c k T_{*}} \frac{1}{r^{2}}$.

This critical value of the density defines a boundary radius for the stellar wind, that we can call "Comptonization" radius,
$R_{\text {Comp }}$, by setting $n_{\text {env }}(R)=n_{\text {Comp }}$ in the continuity equation (see Eq. (3)). We thus obtain

$R_{\mathrm{Comp}}=\left[\frac{\Xi_{\mathrm{c}} \dot{M}_{*} c k T_{*} r^{2}}{m_{\mathrm{H}} v_{0} R_{*}^{-\alpha} L_{\mathrm{ion}}}\right]^{\frac{1}{2+\alpha}}$

In Eqs. (11) and (12) the quantities $T_{*}$ and $\Xi$ cannot be freely chosen, since they depend on each other, their specific relationship, $T_{*}(\Xi)$, deriving from the analysis of possible equilibrium configurations existing in a photoionized gas (Krolik et al. $1981)$. Since $T_{*}(\Xi)$ depends on the assumed continuum radiation illuminating the gas, as well as on other heating mechanisms possibly present and on cooling processes, its resulting detailed shape turns out to be different in literature depending on the specific assumptions of the authors. Krolik (1999) shows a $T(\Xi)$ curve, obtained with the composite continuum spectrum from Elvis et al. (1994), in which $\Xi_{\mathrm{c}} \simeq 10$ and the corresponding cool phase limit temperature is $\sim 5 \times 10^{4} \mathrm{~K}$. In other studies (see Krolik 2002), the "cool" phase $\left(T<10^{5} \mathrm{~K}\right)$ exists for $\Xi \leq 28$ for the continuum spectrum considered by Kaspi et al. (2001), whereas for Krolik \& Kriss (2001) it is present only for $\Xi \leq 12$. The temperature corresponding to these upper limits for the ionization parameter $\Xi$ is around $5 \times 10^{4} \mathrm{~K}$ for the two cases. It is somehow difficult to make a consistent choice for the values of $\Xi_{\mathrm{c}}$ and $T_{*}$ to be used in Eq. (12) without computing directly the specific equilibrium, therefore we choose to rely on the assumptions of one of the papers in literature, namely the one of Kazanas (1989). In this work, the author computes the critical density for the transition to the Comptonized phase (see Eq. (11)) taking the critical value of the ionization parameter as $\Xi_{\mathrm{c}}=10$ and a temperature ( $T_{*}$ in our notation) equal to $3 \times 10^{4} \mathrm{~K}$ (Kazanas 1989). Given the results of most recent studies and calculations (some of which have been cited above), these values can be regarded as sort of "lower limits" for the possible range of critical ionization parameter and/or temperature. However, in the present context we maintain this choice as representative of the critical condition, since, owing to the functional dependence of the limiting radius, $R_{\text {Comp }}$, as expressed in Eq. (12), "lower limit" values for $\Xi_{\mathrm{c}}$ and the corresponding $T_{*}$ guarantee a "lower limit" value for the Comptonization radius as well. In fact, we are evaluating this radius to compare the efficiency of this confinement mechanism with that of the other relevant ones. If indeed Comptonization turned out to be the effective confinement mechanism for the stellar envelopes contributing to the line emission in the BLR, a more accurate analysis of appropriate parameters to be used in the precise evaluation of $R_{\text {Comp }}$ would be required. Nevertheless, if, as it will be proved to be the case in all the conditions of interest for envelopes contributing to the construction of our BLR model, the physical extension of the stellar envelopes is not determined by $R_{\text {Comp }}$, the present evaluation is fully representative. Also, it is important to note that, if this is the case, the Comptonization radius limitation itself is no longer physically relevant to the problem. In fact, outside the otherwise confined stellar envelope, the gas is mixed up with the surrounding nuclear wind and shares its physical properties.

Finally, AN1 mention another limit for the external radius of the stellar wind envelope, deriving from the requirement that 
the stellar wind velocity, when it is increasing outwards, does not reach values larger than $0.1 c$. This is a rather artificial constraint, defining a radius $R_{\mathrm{v}}$, which, for our choice of the expansion velocity of the stellar atmosphere, is $R_{\mathrm{v}}=R_{*}\left(0.1 c / v_{0}\right)^{1 / \alpha}$, and should apply only in extreme cases.

In summary, we define the effective stellar envelope extension at each distance $r$ from the central black hole as the smallest of the various radii determined above:

$R_{\text {ext }}=\min \left[R_{\text {bow }}, R_{\text {tidal }}, R_{\text {mass }}, R_{\text {Comp }}, R_{\mathrm{v}}\right]$.

From our computations, for appropriate BLR models the most efficient confinement mechanism turns out to be the bow-shock formation, thus selecting $R_{\text {bow }}$ as the stellar envelope extension in the equation above. In this case, for larger distances from the star center, i.e. for $R>R_{\mathrm{bow}}$, the gas physical properties are just those of the nuclear wind gas (i.e., $n=n_{\mathrm{w}}$ and $T=T_{\mathrm{w}}$ ), since the shock transition is a sharp one. Using the notation of Eq. (6), the characteristic number density $n_{*}$ can be derived from the continuity equation (Eq. (6) with $R_{\text {ext }}$ the place of $\left.R_{\text {bow }}\right)$

$n_{*}(r)=\frac{\dot{M}_{*}}{4 \pi m_{\mathrm{H}} R_{\mathrm{ext}}^{2} v_{*}}$.

In the current framework, the stellar wind gas generates the line emission via central radiation re-processing, hence the quantities $R_{\text {ext }}$ and $n_{*}$ determine the observed properties of the emission lines. An AGN wind which generates suitable values of the quantities $n_{*}$ and $R_{\text {ext }}$ through Eqs. (14) and (13) can then represent the requested link between the central engine and other structural components of the AGN itself, such as the BLR.

\section{Mass deposition in the AGN wind}

In a picture where the AGN wind expands among mass-losing stars, the presence of the stars will influence the AGN wind dynamics by adding mass to the wind itself. Two different sources of mass deposition are possible: the mass directly lost by the stars and that stripped by star collisions. This implies that nuclear wind models in such a framework must allow for mass deposition increasing the total mass flux along the radial coordinate. Indeed, this possibility has been taken into account in Paper I, in order to maintain the mass density in the wind at non-negligible levels in the outer region, by accounting for a possible mass source along the wind. In this case the total mass flux (mass/time, dimensionally) is not a constant, but is rather a function of the radial coordinate $r$, namely $\dot{M}_{\mathrm{w}}=4 \pi m_{\mathrm{H}} n_{\mathrm{w}} v_{\mathrm{w}} r^{2}=4 \pi A(r)=4 \pi A_{0} f_{\mathrm{m}}(r)$. In this expression, $A(r)$ and the constant $A_{0}$ are dimensionally mass per unit time per unit solid angle, whereas $f_{\mathrm{m}}(r)$ is a dimensionless function of radial distance from the central black hole (see Paper I, Sect. 3 for details). The constant $A_{0}$ is defined as $A_{0} \equiv n_{\mathrm{c}} m_{\mathrm{H}} r_{\mathrm{c}}^{2} c_{\mathrm{sc}}$, where $c_{\mathrm{sc}}$ is the sonic speed at the critical point $r_{\mathrm{c}}$ and $n_{\mathrm{c}}$ is a characteristic value of the wind number density, which is related to the number density value at the critical point by the following relation: $n_{\mathrm{w}}\left(r_{\mathrm{c}}\right)=n_{\mathrm{c}} f_{\mathrm{m}}\left(r_{\mathrm{c}}\right)$. With the notation defined above, the mass source term [mass/time/volume/solid angle $)]$ is $\nabla \cdot\left(m_{\mathrm{H}} n_{\mathrm{w}} \boldsymbol{v}_{\mathrm{w}}\right)=(\mathrm{d} A / \mathrm{d} r) / r^{2}$.
In order to evaluate the amount of mass fed into the wind by stellar mass loss and by star collisions, we have computed the contribution from each process as described in the following. The total contribution, up to a distance $r$ from the central black hole, to the mass flux in the nuclear wind from these external sources is

$\dot{M}_{\text {inp }}=4 \pi\left[A(r)-A\left(r_{0}\right)\right]$,

where $r_{0}$ is the radius at the base of the wind, and, consequently, $4 \pi A\left(r_{0}\right)$ is the "intrinsic" mass flux in the nuclear wind, at its starting point.

Before proceeding, we need to define a schematic description of the spherically symmetric compact dense stellar cluster that we suppose to be located at typical BLR distances from the central black hole of the AGN. In the present work, we assume to represent it in a strongly simplified way, that is considering just three different stellar components for it, main sequence stars (MSs), red giants (RGs), and supergiants (SGs). These three stellar components lose mass to the external interstellar medium, which is here the nuclear wind plasma, both due to their own stellar winds, whose relevance depends of course on the specific stellar component, and because of the possible collision-like encounters that the stars undergo. We also define the relative percentage of RGs and SGs, with respect to the total stellar number density of the cluster, $\rho_{*}$, as $f_{\mathrm{RG}}$ and $f_{\mathrm{SG}}$ respectively, so that $\left(\rho_{*}\right)_{\mathrm{RG}}=f_{\mathrm{RG}} \rho_{*}$ and $\left(\rho_{*}\right)_{\mathrm{SG}}=f_{\mathrm{SG}} \rho_{*}$, whereas, for reasonably small values of the fractions $f_{\mathrm{RG}}$ and $f_{\mathrm{SG}}$, we can take $\left(\rho_{*}\right)_{\mathrm{MS}} \simeq \rho_{*}$, i.e., $f_{\mathrm{MS}} \simeq 1$. In the computation of our models, we have usually assumed a "standard" value for the relative fractions of RG and SG stars, that is $f_{\mathrm{RG}}=0.01$ and $f_{\mathrm{SG}}=10^{-4}$ (see AN1), but we keep those fractions generically as parameters of the problem in what follows.

As for the stellar mass loss from the stars of a central cluster characterized by a stellar number density $\rho_{*}(r)$ and a composition as defined above, the total contribution to the AGN nuclear wind mass loading rate, up to a radial distance $r$ can be expressed as

$\dot{M}_{* \text { wind }}(r) \simeq$

$\int_{r_{0}}^{r}\left\{\left(\dot{M}_{*}\right)_{\mathrm{MS}} f_{\mathrm{MS}}+\left(\dot{M}_{*}\right)_{\mathrm{RG}} f_{\mathrm{RG}}+\left(\dot{M}_{*}\right)_{\mathrm{SG}} f_{\mathrm{SG}}\right\} \rho_{*} 4 \pi r^{2} \mathrm{~d} r$,

where $\left(\dot{M}_{*}\right)_{i}$ represents the mass loss rate in the wind of the " $i$ "th stellar component of the cluster. For reasons that will be extensively discussed in the next sections and are related to the very specific and extreme environment in which the central stellar cluster is located (see, for example, Taylor 1999), here we allow for a possible non-negligible contribution from main sequence (MS) stellar winds.

As already mentioned, the other contribution to mass deposition into the AGN nuclear wind comes from stellar collisions. Following AN1 and Murphy et al. (1991), we only take into account collisions between stars of the same type, thus neglecting the contribution due to MS-RG and MS-SG star collisions, which are characterized by a much smaller fractional mass loss with respect to collisions between same type stars. In the hypothesis that for each collision a fraction $f_{\mathrm{c}}$ (that we take to be 0.1 following AN1 and Begelman \& Sikora 1992) of one solar mass of matter is stripped from the colliding stars and fed 
into the wind, the total mass deposition rate due to collisions occurred up to radial distance $r, \dot{M}_{* \text { coll }}(r)$, is

$$
\begin{aligned}
& \dot{M}_{* \mathrm{coll}}(r) \simeq \\
& 0.1\left[\left(\tau_{\text {coll }}\right)_{\mathrm{MS}}(r)+\left(\tau_{\text {coll }}\right)_{\mathrm{RG}}(r)+\left(\tau_{\text {coll }}\right)_{\mathrm{SG}}(r)\right] M_{\odot} / \mathrm{yr},
\end{aligned}
$$

where $\left(\tau_{\text {coll }}\right)_{i}$ is the star collision rate (here expressed as number of collisions per year) for the stellar component " $i$ " defined as:

$$
\left(\tau_{\text {coll }}\right)_{i}(r)=4 \pi^{2}\left(R_{*}\right)_{i}^{2} \int_{r_{0}}^{r}\left(f_{i} \rho_{*}\right)^{2} r^{2} V(r) \mathrm{d} r
$$

with $\left(R_{*}\right)_{i}$ the stellar radius of stars of type " $i$ ", and $f_{i}$ the fraction of stars of type " $i$ ", that is assumed to be equal to unity for the case " $i "=\mathrm{MS}$, that is for the main sequence star component.

For the definition given above of $\dot{M}_{\text {inp }}(r)$, the following relationship must hold

$$
\dot{M}_{\text {inp }}(r)=\dot{M}_{* \text { wind }}(r)+\dot{M}_{* \text { coll }}(r)
$$

Indeed, we are interested in the equation that can be obtained from the previous one by deriving it with respect to $r$ and dividing by $4 \pi$, and that represents the equation for the sum of the mass sources from stellar matter expulsion processes at distance $r$, giving the total mass source function per unit solid angle $(\mathrm{d} A(r) / \mathrm{d} r) / r^{2}=\left(A_{0} / r^{2}\right) \mathrm{d} f_{\mathrm{m}} / \mathrm{d} r$ for the nuclear wind (see Paper I). This equation turns out to be the following:

$A_{0} \frac{\mathrm{d} f_{\mathrm{m}}(r)}{\mathrm{d} r} \frac{1}{r^{2}}=F(r)$

where the function $F(r)$ is defined as follows

$$
\begin{aligned}
F(r) & =\left[\left(\dot{M}_{*}\right)_{\mathrm{MS}} f_{\mathrm{MS}}+\left(\dot{M}_{*}\right)_{\mathrm{RG}} f_{\mathrm{RG}}+\left(\dot{M}_{*}\right)_{\mathrm{SG}} f_{\mathrm{SG}}\right] \\
& \times \rho_{*}(r)+0.1 M_{\odot} \pi V(r) \rho_{*}^{2}(r) \\
& \times\left[\left(R_{*}^{2}\right)_{\mathrm{MS}} f_{\mathrm{MS}}^{2}+\left(R_{*}^{2}\right)_{\mathrm{RG}} f_{\mathrm{RG}}^{2}+\left(R_{*}^{2}\right)_{\mathrm{SG}} f_{\mathrm{SG}}^{2}\right] .
\end{aligned}
$$

From Eqs. (20) and (21), given $\rho_{*}(r)$, it is evident that for a given choice of the physical parameters of each stellar component, $A(r)$ can be derived by solving the equation itself. As it will be discussed in a following section, the prescription of $\rho_{*}(r)$ is constrained by several factors, but $\rho_{*}(r)$ must be chosen so as to obtain a mass source function with a sufficiently smooth behaviour. This is relevant for the successful integration of the nuclear wind equations. Of course, the resulting mass source function depends on the mass loss rates that we adopt for our model cluster stars, as well as on the chosen values of $\left(R_{*}\right)_{i}$, and of the population fractions $f_{i}$, for the three stellar components of the cluster; this is also extensively discussed in the following sections.

A numerical comparison of the two terms on the right hand side of Eq. (21) can show whether one of the two processes of mass deposition in the nuclear wind predominates and, in that case, which one it is, as a function of $r$. For a given $\rho_{*}(r)$, the result of this analysis strongly depends on the choice of the mass loss rates of the various types of stars in the model cluster.

\section{Model parameters}

\subsection{Free parameters in the model}

As it is apparent from the discussions in Sects. 2 and 3, in order to derive the effective stellar envelope extension and its density through Eqs. (14) and (13), the wind model of Paper I must be extended to take into account the mass deposition function (as derived from Eqs. (20) and (21)) and to include the computation of $R_{\text {ext }}(r)$ and $n_{*}(r)$. This process implies the introduction of a number of parameters that can be divided in three classes, each associated with a different aspect of the problem. These parameters are summarized in the following for the sake of clarity. As described below, some of these parameters have almost no influence on the final results, some have been fixed to standard values and some other represent the control keys to variations in the outcome of our model.

a) Parameters related to the AGN nucleus and its wind: central radiation field luminosity, $L$, characteristic number density, $n_{\mathrm{c}}$, and temperature, $T_{\mathrm{c}}$, of the wind plasma at the critical point (that is at the distance at which the wind outflow changes its character from subsonic to supersonic). For a given luminosity the choice of $T_{\mathrm{c}}$ does not affect the results since a different temperature value essentially shifts the critical point position leaving all physical quantities profiles almost unaffected. We have shown in Paper I that the characteristic wind number density, $n_{\mathrm{c}}$ (related to the number density at the critical point, as defined in Sect. 3) is a critical parameter, and the system is very sensitive to its variations. Indeed, a solution for the nuclear wind equations is possible and characterized by a total electron scattering optical depth of the wind plasma $\lesssim 1$ (see Paper I) only over a rather narrow range of values for $n_{\mathrm{c}}$. Variations of $n_{\mathrm{c}}$ within this range could in principle affect the BLR model and consequently might change the emission line properties; we discuss this issue in Sect. 5.

b) Parameters characterizing the nature of the stellar cluster orbiting around the nucleus: star number density distribution $\rho_{*}(r)$, mass-loss rate and radius $\left(\dot{M}_{*}, R_{*}\right)$ for each type of star (SG, RG, MS), and percentage of each type of stars present in the cluster ( $f_{i}$ for stellar type " $i$ "). These parameters enter the computation of stellar envelope properties, but also contribute to determine the mass deposition function $f_{\mathrm{m}}(r)$ and hence influence the nuclear wind integration (see Sect. 3). Some parameters have been fixed to their "standard" values. This is the case for the star percentages and radii which are set as $f_{\mathrm{SG}}=10^{-4}$, $f_{\mathrm{RG}}=10^{-2}, f_{\mathrm{MS}} \sim 1$ and $\left(R_{*}\right)_{\mathrm{SG}}=100 R_{\odot},\left(R_{*}\right)_{\mathrm{RG}}=10 R_{\odot}$, $\left(R_{*}\right)_{\mathrm{MS}}=1 R_{\odot}$. For others, namely $\rho_{*}(r)$ and $\left(\dot{M}_{*}\right)_{i}$, different possibilities have been analysed. In particular for stellar mass loss rates the assumed values and their influence on the final results are described in the following sections.

As far as the star number density distribution is concerned, there is no observational information about its detailed profile in regions so close to the central nucleus, the only possible indications come from theoretical work. Therefore, we have chosen $\rho_{*}(r)$ profiles in accordance with two main suggestions derived from the results of David et al. (1987a, 1987b) analyses of a star cluster evolution around a black hole. The first suggestion regards the peak value for the star number density in an 

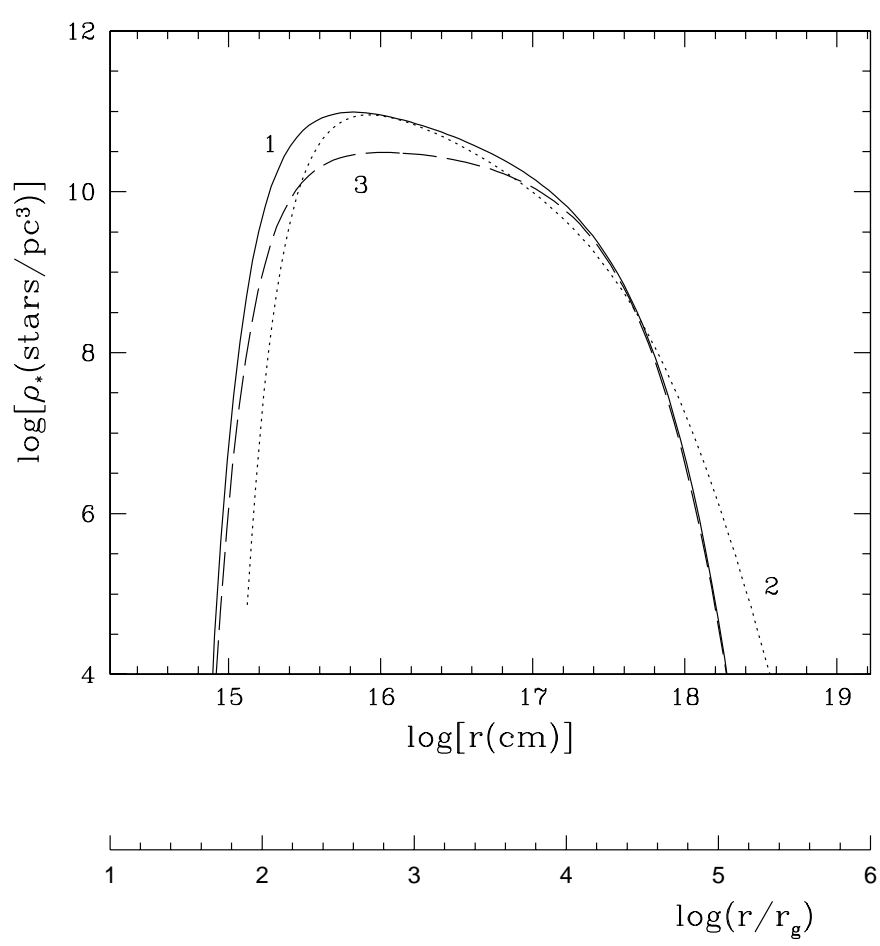

Fig. 1. Star number density as a function of the distance $r$ from the AGN nucleus. We also show the abscissa axis for the corresponding dimensionless distance $x=r / r_{\mathrm{g}}$ for the case of a central black hole of mass $5.6 \times 10^{7} M_{\odot}$, that we have chosen to associate to a central luminosity $L=10^{44} \mathrm{erg} / \mathrm{s}$ (see Paper I). Label meaning is explained in the text.

evolved cluster, that turns out to be around $10^{11} \mathrm{stars} / \mathrm{pc}^{3}$. The second one is that an evolved star distribution is concentrated in a range of distances from $10^{14} \mathrm{~cm}$ up to $10^{18} \mathrm{~cm}$ from the central black hole and steeply decreases both for shorter and for longer distances. We have analysed several different $\rho_{*}(r)$ profiles obeying these criteria, and present here the results for the stellar distribution profiles that seem to best represent our working scenario. The selected curves are shown in Fig. 1. In this figure the curve labeled " 1 " is representative of the class of star density distribution which gives the best results for our model. All the results presented in the following sections refer to this particular choice of $\rho_{*}(r)$. The other two curves have been illustrated since an analysis of the consequent results allows us to highlight separately the effects of increasing $\rho_{*}(r)$ in the external region (curve " 2 ") and those of decreasing $\rho_{*}(r)$ in the intermediate region (curve " 3 ").

Possible differences in the stellar distribution profiles in the region $r<2 \times 10^{15} \mathrm{~cm}$ have proved to be unimportant in the present context, owing to the fact that our integration of the nuclear wind equations stops at around that distance. We elected to retain the same profiles $\rho_{*}(r)$ as shown in Fig. 1, when analyzing configurations with different central black hole masses (that we associate with a different central luminosity, see Paper I), thus implying different values for $r_{\mathrm{g}}$, and, consequently different resulting profiles as functions of the dimensionless distance $x \equiv r / r_{\mathrm{g}}$.

c) Parameters defining the physical properties of the stellar envelopes: stellar wind velocity profile (see Eq. (2)) and temperature value $\left(v_{0 i}, \alpha_{i},\left(T_{*}\right)_{i}\right)$, for each type of star ("i" " $=\mathrm{SG}$, $\mathrm{RG}, \mathrm{MS})$. This group of parameters only influences the properties of the line-emitting regions.

In summary, once we have chosen the luminosity of the AGN for which we want to build up a model, the free parameters we can work with turn out to be the following quantities: $n_{c}, \rho_{*}(r),\left(\dot{M}_{*}\right)_{i}, v_{0 i}, \alpha_{i}$ and $\left(T_{*}\right)_{i}$. The "observational requirements" on the BLR described in the following subsection dictate further limitations to the number of parameters that significantly influence the model.

\subsection{BLR parameters and observational requirements}

Not every combination of the above listed free parameters can reproduce the observed BLR general properties. Observative and interpretative work has shown that definite ranges of parameters and/or quite specific features are common to the BLRs of most AGNs. In this section these observational requirements are "translated" in terms of our model variables.

From the observational and interpretative points of view, our spherical stellar atmospheres can contribute to the BLR emission only if $R_{\mathrm{ext}}(r)$ and $n_{*}(r)$ satisfy the following conditions:

a) $R_{\text {ext }}(r) \geq \Delta R=10^{23} U / n_{*} \mathrm{~cm}$, since photoionization equilibrium requires that emission lines are generated in shells of thickness $\Delta R$, which is at least $\Delta R=10^{23} U / n_{*} \mathrm{~cm}$ (see e.g. Peterson 1997). The definition of an emitting shell then allows an estimate of the mean density of the emitting plasma in the shell itself. Since the density inside the stellar expanding envelope varies as shown in Eq. (3), the mean density can be evaluated as

$\hat{n}_{*}(r)=\left(\frac{\dot{M}_{*}}{4 \pi m_{\mathrm{H}} v_{0} R_{*}^{2}}\right) \int_{R_{\mathrm{ext}}-\Delta R}^{R_{\mathrm{ext}}}\left(\frac{R}{R_{*}}\right)^{-(\alpha+2)} \frac{\mathrm{d} R}{\Delta R}$,

where both the integration limits and possibly the mass loss rate depend on $r$. In terms of the quantity just defined, the other conditions a BLR emitting plasma must satisfy read:

b) $\hat{n}_{*}(r) \geq 10^{8} \mathrm{~cm}^{-3}$, since broad forbidden lines are not observed (Netzer 1990; Peterson 1997);

c) if the line width is due to thermal effects only, it must be $\hat{n}_{*}(r)<1 \times 10^{12} \mathrm{~cm}^{-3}$ to avoid line thermalization, i.e., for line emission to be effectively significant (see Rees et al. 1989; Kaspi \& Netzer 1999; Korista \& Goad 2000).

In our model $R_{\text {ext }}(r)$ and $\hat{n}_{*}(r)$ will attain different values at different distances from the central black hole, hence conditions a), b) and c) will be satisfied only in a specific range of distances. We call $r_{1}$ and $r_{2}$ the distances which delimit this interval. Stellar envelopes satisfying points a) to c) contribute to build up the total covering factor. Here we define

$C_{i}(r)=\int_{\left(r_{1}\right)_{i}}^{r} \pi\left(R_{\mathrm{ext}}\right)_{i}^{2} f_{i} \rho_{*} \mathrm{~d} r$

as the covering factor accumulated at distance $r$ from the central black hole due to contributions from star type " $i$ " (" $i$ " $=S G$, RG, MS), obtained using $R_{\text {ext }}(r)$ computed for that specific star type. To obtain the total contribution to the covering factor due to a given stellar type, in Eq. (23) we have to integrate up to 
$r=r_{2}$, i.e. up to the external boundary of the interval defining the broad line contributing region for each stellar type, that is we have to compute $C_{i}\left(r_{2}\right)$ for " $i$ " $=\mathrm{MS}, \mathrm{RG}, \mathrm{SG}$. Finally, we define the total covering factor $C_{\text {tot }}$ for a given BLR model as the sum of the total contributions, that is

$C_{\mathrm{tot}} \equiv C_{\mathrm{MS}}\left(r_{2}\right)+C_{\mathrm{RG}}\left(r_{2}\right)+C_{\mathrm{SG}}\left(r_{2}\right)$.

Our model can now be compared with observations, testing the following points:

1) reasonable values should be recovered for the covering factor $C_{\text {tot }}$, whose estimates range from about 0.05 up to 0.25 (Baldwin 1997);

2) the typical distance characterizing the BLR, namely $r_{\mathrm{BLR}}$ (see Sect. 1) must be contained in the intervals $\left[r_{1}, r_{2}\right]_{i}$ relative to the stellar types contributing to the definition of the value of $C_{\text {tot }}$;

3 ) the covering factor relative to "broad" forbidden emission lines, $C_{\text {forb }}$, must be vanishing. In our model, we define

$C_{\text {forb }}=\sum_{i}\left(C_{\text {forb }}\right)_{i}=\sum_{i} \int_{r_{0}}^{\infty} \pi\left(\hat{R}_{\text {ext }}\right)_{i}^{2} f_{i} \rho_{*} \mathrm{~d} r$

where

$\left(\hat{R}_{\mathrm{ext}}\right)_{i}=\left\{\begin{array}{lll}\left(R_{\mathrm{ext}}\right)_{i} & \text { if } & n_{*}\left[\left(R_{\mathrm{ext}}\right)_{i}\right]<10^{8} \mathrm{~cm}^{-3} \\ 0 & \text { if } & n_{*}\left[\left(R_{\mathrm{ext}}\right)_{i}\right] \geq 10^{8} \mathrm{~cm}^{-3}\end{array}\right.$

(see Peterson 1997; Krolik 1999), therefore, a contribution to $C_{\text {forb }}$ comes only from those envelopes whose photoionized shell interior to the confining bow shock has a density appropriate for forbidden line emission.

4) the ionization parameter should be in in the range 0.01-1. Assuming $<h v>=2.7 \mathrm{Ryd}$ as in AN1, and with $L$ in $\mathrm{erg} / \mathrm{s}, \hat{n}_{*}$ in $\mathrm{cm}^{-3}$ and $r$ in $\mathrm{cm}$, the expression for $U$ shown in the Sect. 1 becomes

$U(r)=4.5 \times 10^{-2} \frac{L}{\hat{n}_{*} r^{2}}$.

The above general requirements allow us to evaluate the validity of our model and to restrict the range in which free parameters described in the above subsection can vary.

\section{Model results}

In Paper I, we have defined a model for an AGN nuclear wind, that turned out to be essentially determined by the choice of the central radiation field luminosity, $L$, of $n_{\mathrm{c}}$, the characteristic number density of the wind plasma (see Sect. 4.1), and of the mass deposition function $f_{\mathrm{m}}(r)$ (see Sect. 3 and Paper I). As it is discussed in Sect. 3, the mass source function (and therefore ultimately $f_{\mathrm{m}}(r)$ ) for the nuclear wind depends on the choice of both the star number density distribution of the central cluster, $\rho_{*}(r)$, and the star cluster composition and properties (such as mass loss rates, radii and percentages of the three different star types that we assume as components of the stellar population of the cluster; see Sect. 4.1 for details). As a consequence, the choice of all the cluster parameters mentioned above strongly influences the general properties of the solution for the nuclear wind as well. These, in turn, enter the determination of the physical quantities characterizing the stellar envelopes that we consider to be the line emitting sources.

The above described complex interplay between star and interstellar medium properties makes it difficult to predict our model results. While it is obvious that the validity of our model could be proved only by the detailed comparison of predicted and observed line profiles, it seems of primary importance to understand which are the relevant parameters in our picture. Hence, due to the large number of parameters and the complexity of the model, a general analysis aimed at performing a first selection among all the present parameters is preferable, in order to get a deeper insight into the flexibility of the model itself. Therefore, as a first step in the comparison between observed BLR features and our model results, we have analysed in detail the influence that each free parameter has in the fulfillment of the observational requirements presented in Sect. 4.2. The results of this analysis are presented hereafter. We refer to Sect. 4.1 for the specific definition of the values chosen and fixed as "standard" for stellar radii and percentages in the cluster.

Effects of changing the star density distribution $\rho_{*}$.

As we have mentioned in Sect. 4.1, point $b$ ), our system is very sensitive to the choice of $\rho_{*}$. Indeed, the results obtained by using the three stellar distributions shown in Fig. 1 turn out to be significantly different. The "small" differences among $\rho_{*}$ profiles are sufficient to exclude the star distribution labeled with " 2 ". In fact, any stellar distribution $\rho_{*}(r)$ that is more extended in the "external" region (i.e., for $r \gtrsim 10^{18} \mathrm{~cm}$ ) than the one characterized by profile " 1 ") can reproduce a covering factor in accordance with point 1 ) of Sect. 4.2, but also shows a resulting non-negligible forbidden line covering factor, so that it cannot satisfy point 3) of Sect. 4.2. On the other hand, for profiles similar to the one labeled with "3", i.e. characterized by a smaller amount of stars, the forbidden covering factor is negligible, but $C_{\text {tot }}$ turns out to be too low with respect to the range of values inferred from observations if suitably high values of stellar mass loss rates are not used. This is apparent from Eq. (23), taking into account that $R_{\text {ext }}^{2}$ is generally proportional to the ratio $\dot{M} / v_{0}$ (see Eq. (7)). Accounting for the considerations above, in the following we have chosen to discuss the results pertaining to the the star number density profile labeled with "1" in Fig. 1, as representative of a possible cluster star distribution for broad line emitting AGNs.

Effects of changing the nuclear wind density parameter $n_{\mathrm{c}}$.

As shown in Paper I (Eqs. (14) and (15)), the AGN wind integration system depends on the quantity $f_{\mathrm{m}}^{\prime} / f_{\mathrm{m}}$, which can be easily derived as

$\frac{f_{\mathrm{m}}^{\prime}}{f_{\mathrm{m}}}=\frac{r^{2} F(r)}{A_{0} f_{\mathrm{m}}\left(r_{0}\right)+\int_{r_{0}}^{r} r^{2} F(r) \mathrm{d} r}$

where it is $f_{\mathrm{m}}^{\prime}=r^{2} F(r) / A_{0}$ from Eq. (20). Here the parameters of the star cluster define the mass source function per unit solid angle, that is the function $F(r)$, through Eq. (21) and $f_{\mathrm{m}}\left(r_{0}\right)$ is an integration constant. Once chosen a specific model, i.e. for fixed $F(r)$, the relative weight of the terms $A_{0} f_{\mathrm{m}}\left(r_{0}\right)$ and $\int_{r_{0}}^{r} r^{2} F(r) \mathrm{d} r$ defines the amount of of mass flux characterizing the nuclear wind at its base with respect to that fed by stars. 
Hence, changing $n_{\mathrm{c}}$, which enters the definition of $A_{0}$, changes the function $f_{\mathrm{m}}^{\prime} / f_{\mathrm{m}}$ mainly in the "internal" region $\left(r \sim r_{0}\right)$ where the term $A_{0} f_{\mathrm{m}}\left(r_{0}\right)$ is non-negligible with respect the integral term. Increasing $n_{\mathrm{c}}$, the intrinsic mass flux of the nuclear wind becomes more important with respect to the mass flux fed by stars and the electron scattering optical depth may become too large (as discussed in Paper I). For $r>>r_{0}$, in the region where the BLR is located, the integral $\int_{r_{0}}^{r} r^{2} F(r) \mathrm{d} r$ is the predominant term in the mass flux definition and a change in the critical density value does not have a significant effect on the final result. A numerical analysis confirms the present analytical discussion, therefore we conclude that, once $n_{\mathrm{c}}$ is chosen in the restricted range allowed by the nuclear wind integration (as explained in Sect. $4.1 \mathrm{a}$ ), its changes do not affect the BLR properties as derived from our model.

Effects of changing the velocity profile of the stellar wind, i.e. the value of $\alpha$, in Eq. (2).

Several integrations of our model, carried on with different $\alpha$ values, have shown that the specific velocity profile does not have a strong influence on the results. However, positive $\alpha$ values are generally preferable, since the corresponding models are characterized by lower values of the forbidden line covering factor with respect to those with negative $\alpha$ values. Perhaps, the most straightforward way to understand this is to go along the following line of reasoning. When the stellar envelope extension is delimited by the bow shock (which is the case under most of the conditions of physical interest), the density of the envelope at its boundary, $n_{*}$, is determined by the local density of the AGN nuclear wind plasma, since $n_{*} \propto n_{\mathrm{w}}$, as it can be realized by inserting Eqs. (7) in (6). This implies that, going farther from the AGN central black hole, $n_{*}$ decreases, like $n_{\mathrm{w}}(r)$ does. Accounting for this condition in Eq. (6), it is easy to see that, for a given $\dot{M}, R_{\text {ext }}$ increases with $r$, and it increases faster for negative values of $\alpha$. As a consequence of this last point, for negative $\alpha$ values non-negligible contributions to the covering factor are expected to come from regions that are far enough (in the computations that we present in the following subsections, this is generally for $r \gtrsim 2-3 \times 10^{4} r_{\mathrm{g}}$ ) from the center of the AGN, so that the stellar envelope density is sufficiently low to be suitable for forbidden line emission.

\section{Effects of changing the star envelope temperature.}

Values of the temperature in the range $1-3 \times 10^{4} \mathrm{~K}$, appropriate for BLR emission, have been tested, obtaining no significant change in the results. This fact supports our simplifying assumption of a constant temperature profile for the overall stellar expanding envelope.

Taking into account all the above points, we conclude that in our BLR model only a specific class of star number density profiles meets the observational requirements, while changes in the values of the parameters $n_{\mathrm{c}}, \alpha$ and $T_{*}$ do not have a relevant influence on the quality of the reproduction of BLR properties. For this reason, in the following we illustrate the general results of our exploration of physically reasonable ranges of the parameters whose changes turned out from our analysis to significantly affect the results as for BLR global properties, namely stellar mass loss rates and expansion velocity of the stellar atmospheres (see end of Sect. 4.1). To do this, we have chosen to discuss separately three different choices for the definition of the mass loss rates in the envelopes of the different stellar types composing the cluster, and to examine the effects of changing the expansion velocity of the envelopes on a significant quantity such as the resulting covering factor of the proposed BLR model. Before entering the details of the discussion, we just want to remind that all the results that we show and comment in the following, unless otherwise specified, refer to a specific choice for

(i) the central source luminosity, $L=10^{44} \mathrm{erg} / \mathrm{s}$;

(ii) the black hole mass, $M_{\mathrm{BH}}=5.6 \times 10^{7} M_{\odot}$ (thus defining $r_{\mathrm{g}}$ );

(iii) the envelope temperature, $T_{*}=2 \times 10^{4} \mathrm{~K}$ (see Sect. 4.2);

(iv) the expansion velocity power law exponent, $\alpha=0.75$ (see Sect. 4.2);

(v) the star number density, $\rho_{*}(r)$, labeled "1" in Fig. 1,

where choices (iii), (iv) and (v) are justified by the above discussion.

As for the stellar mass loss rates, we have chosen to discuss

a) the simple case of "standard" mass loss rates for stellar winds of stars of a given type, as known from galactic studies (see below for the details and values, Sect. 5.1);

b) a case in which we try to account for the possibility of "enhanced" mass loss of MS, RG, and SG stars, due to the very specific context in which they are set, namely a strong X-ray illumination from the central source in the AGN (see Sect. 5.2); this would lead to an expected enhancement of mass loss rates increasing with decreasing $r$, with a behaviour following the radiation flux increase going inwards, that is $\propto r^{-2}$;

c) a third case in which we suppose the mass loss rates do again depend on the distance from the central black hole $r$, but with a flatter increase going inwards (Sect. 5.3). The physical mechanism responsible for this configuration is not specified in this case, but we refer to Sect. 6 for a brief discussion of possible processes.

\subsection{Standard mass loss rates}

The first reasonable step is that of assuming "standard" mass loss rates for the stars in the cluster (see, for example, Lamers $\&$ Cassinelli 1999), that is

$\left(\dot{M}_{*}\right)_{\mathrm{SG}} \simeq 10^{-6} M_{\odot} / \mathrm{yr}$

$\left(\dot{M}_{*}\right)_{\mathrm{RG}} \simeq 10^{-8} M_{\odot} / \mathrm{yr}$

$\left(\dot{M}_{*}\right)_{\mathrm{MS}} \simeq 10^{-14} M_{\odot} / \mathrm{yr}$.

With this choice for the mass loss rates of the stars in the central cluster, from Eq. (20) it turns out that, in the inner region of the cluster (i.e., for $r \lesssim 10^{4} r_{\mathrm{g}}$ ), the mass flux input in the nuclear wind is strongly dominated by collisional effects (i.e., mass loss from collisions between stars). On the contrary, farther from the AGN center there is a vast region in which predominance of the stellar wind mass loss sets on. We then derive the corresponding AGN nuclear wind model, and, consistently, the main physical parameters of the BLR emitting envelopes.

For each stellar type these are: i) the evaluation of the characteristic extension for the expanding envelope, $R_{\text {ext }}$ 
(see Eq. (13)), and the physical mechanism defining it; ii) the average density of the envelope over the emitting region of the envelope itself, $\hat{n}_{*}$, (see Eq. (22)); iii) the resulting ionization parameter $U$ (Eq. (25)); iv) the covering factor, as a function of the distance $r$ from the black hole in the interval $\left[r_{1}, r_{2}\right]$ defining the BLR (see Eq. (23)). A typical behaviour (which, we want to stress, is generally common to the three cases for stellar mass loss rates that we present in this work) corresponds to an increasing envelope extension, and to a decreasing density with $r$ increasing. Of course, the covering factor $C_{i}(r)$ is an increasing function of $r$ over the model BLR range of distances, since the contributions from the stars in each shell at a given distance from the black hole accumulate in the integral defined by Eq. (23).

First, we want to focus our attention on the results we obtain from the model in terms of global parameters of the BLR, such as the integrated covering factor, defined as $C_{i}\left(r_{2}\right)$ for each stellar type " $i$ ". We briefly describe the $r$ dependence of the first three physical quantities mentioned above later on in the present section and in Sect. 6 .

The general outcome for this case is exemplified in Fig. 2, showing the resulting covering factor contributions (computed from Eq. (23) with $r=r_{2}$ ) for the modeled BLR, as functions of the terminal velocity $v_{\text {term }} \equiv v_{*}\left(R_{\text {ext }}\right)$, characterizing the stellar envelopes that are assumed to contribute to the broad line emission. We also plot the sum of the contributions due to the different stellar types composing our model central cluster as $C_{\text {tot }}$, again as a function of $v_{\text {term. }}$. We obtain the variation of $v_{\text {term }}$ for each stellar type, by varying $\left(v_{0}\right)_{i}$, which, in turn, also induces variations in the extension of the envelope, at least when the confinement mechanism for the envelope itself is the bow shock (see Sect. 2, Eqs. (7) and (12)); changing this parameter is therefore significant to the determination of the covering factor. To give an idea of the range of $\left(v_{0}\right)_{i}$, we report here the values corresponding to the terminal velocities $v_{\text {term }}=0.1$ and $2.1 \mathrm{~km} \mathrm{~s}^{-1}$, which are close to the extremes of the terminal velocity range plotted in Fig. 2 and representative of "low" and "high" terminal velocities respectively. In the present case, for the star types that do give contribution to the covering factor, the values of $v_{0}$ are $\left(v_{0}\right)_{\mathrm{RG}}\left(v_{\text {term }}=\right.$ $\left.0.1 \mathrm{~km} \mathrm{~s}^{-1}\right)=1.6 \times 10^{-3} \mathrm{~km} \mathrm{~s}^{-1},\left(v_{0}\right)_{\mathrm{SG}}\left(v_{\text {term }}=0.1 \mathrm{~km} \mathrm{~s}^{-1}\right)=$ $3.7 \times 10^{-3} \mathrm{~km} \mathrm{~s}^{-1}$, and $\left(v_{0}\right)_{\mathrm{RG}}\left(v_{\mathrm{term}}=2.1 \mathrm{~km} \mathrm{~s}^{-1}\right)=1.8 \times$ $10^{-2} \mathrm{~km} \mathrm{~s}^{-1},\left(v_{0}\right)_{\mathrm{SG}}\left(v_{\text {term }}=2.1 \mathrm{~km} \mathrm{~s}^{-1}\right)=4.2 \times 10^{-2} \mathrm{~km} \mathrm{~s}^{-1}$.

It is important to note that the plotted value of $C_{\text {tot }}$ is the one that we obtain when we suppose that the three different star type envelopes are endowed with the same value of the terminal velocity, which is not necessarily the case. In fact, one can obtain different values for the total covering factor for the BLR model, with respect to those that we present in the plot, by assuming different $v_{\text {term }}$ values for each single species of stars, that is picking contributions from the various star types at different $v_{\text {term }}$ along the curves defining the specific covering factor for a given stellar type. However, we want to stress that, due to the fact that the profiles $C_{i}\left(v_{\text {term }}\right)$ for the three stellar types show a similar behaviour, which is generally decreasing for increasing $v_{\text {term }}$ (apart from the very low velocity end of the range shown in the figures), it turns out that for any chosen value of $v_{\text {term }}$ the total covering factor we plot in Fig. 2

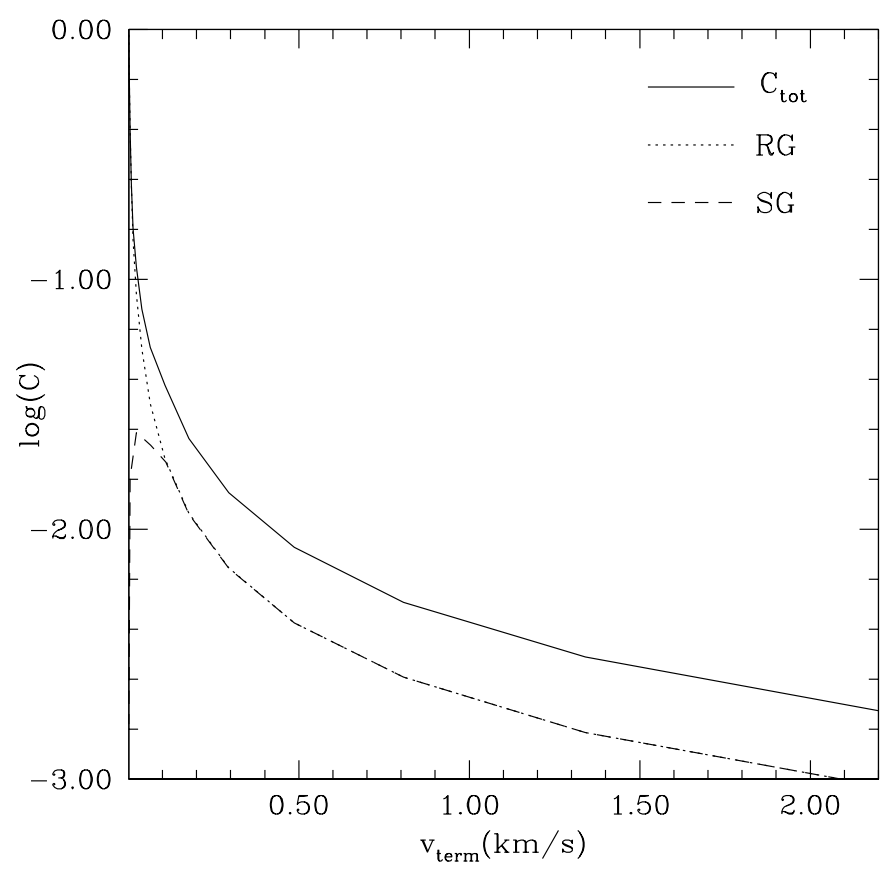

Fig. 2. The total value of the covering factor, $C_{\text {tot }}$, defined as the sum of the contributions due to the envelopes of different stellar types, as well as the distinct contributions to this value from the various stellar types, are shown as functions of the terminal velocity of expansion $v_{\text {term }}$ (in $\mathrm{km} \mathrm{s}^{-1}$ ), characterizing the contributing envelopes. The present figure refers to the case for "standard" mass loss rates. Notice that the contribution due to main sequence stars in this "standard" case does not even appear in the plot, since it is absolutely negligible with respect to those due to RG and SG stars.

is the maximum value that can be attained if we suppose that the different contributing stellar type envelopes are characterized by terminal velocity values that are possibly different, but in any case larger than the one chosen. On the contrary, larger $C_{\text {tot }}$ values can be obtained by allowing for different terminal velocities of different stellar type envelopes, provided the $v_{\text {term }}$ value of at least one of the contributing types is lower than the one chosen for the exemplification. These are general considerations and they refer both to the present and to the other two cases we discuss in the following subsections.

An inspection of the solutions for the BLR physical parameters shows that the bow shock mechanism is in general the most efficient in confining the stellar envelopes and, consequently, determines their extension for all the three different stellar types. As a consequence, for this specific choice of the stellar mass loss rates, the envelope radii for RG stars and SG stars turn out to scale so as to give essentially the same contribution to the total covering factor for the BLR. As it is apparent from Fig. 2, this is no longer true for low velocities $\left(\lessgtr 0.12 \mathrm{~km} \mathrm{~s}^{-1}\right)$, when the contribution to the covering factor due to SG stars "decouples" from that of RGs. The physical reason for this is in the fact that at these low velocities the most efficient confining mechanism for SGs all through the BLR extension becomes tidal disruption of the envelope beyond a given radius (see Eq. (8)), whereas for RGs the bow shock mechanism is still dominant. 


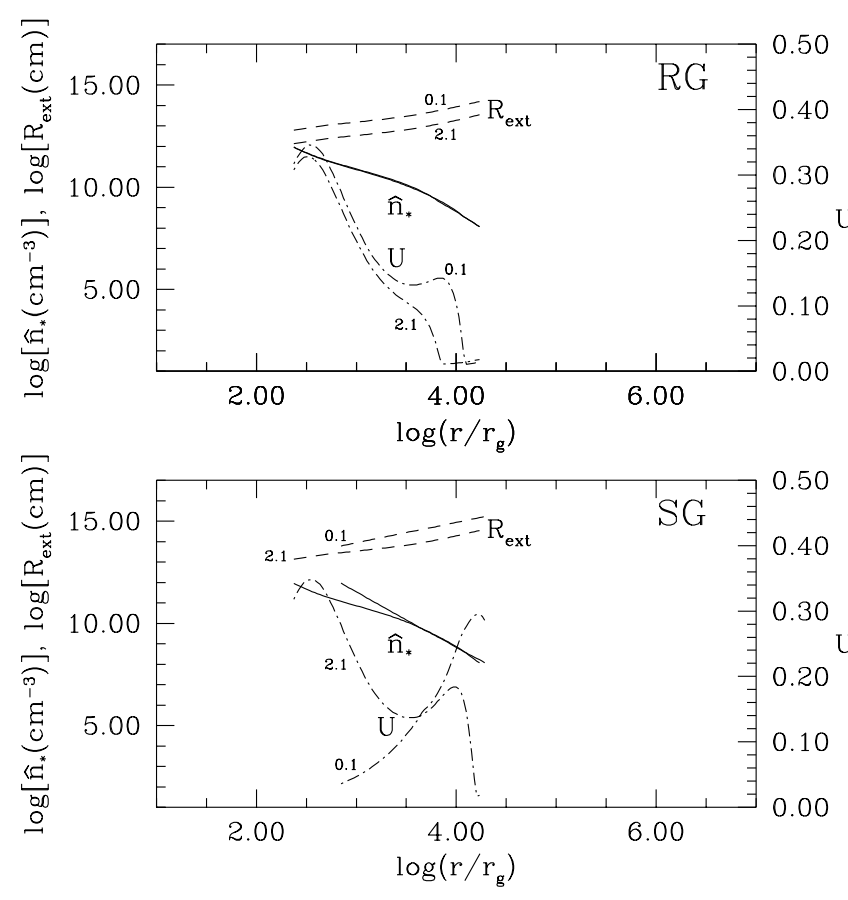

Fig. 3. Physical parameters characterizing stellar envelopes contributing to the BLR as functions of the normalized distance $r / r_{\mathrm{g}}$ from the central black hole; these are the mean density of the envelope, $\hat{n}_{*}(r)$, the estimate of the envelope extension, $R_{\mathrm{ext}}(r)$, and the ionization parameter, $U(r)$. The interval over which the physical quantities are plotted represents the "BLR" interval $\left[r_{1}, r_{2}\right]$ as defined in Sect. 4.2; the labels 0.1 and 2.1 identify the specific value (in $\mathrm{km} \mathrm{s}^{-1}$ ) of the terminal velocity parameter for which they are computed. The upper panel refers to RGs' envelopes, while the lower one shows the results pertaining to the envelopes of SG stars, in the case of "standard" mass loss rates.

To illustrate the behaviour of the BLR physical quantities and the characteristic extension of our BLR model (i.e., the $\left[r_{1}, r_{2}\right]$ interval over which conditions a) to c) of Sect. 4.2 for the emitting gas structures are fulfilled), we have chosen to plot in the two panels of Fig. 3 (each one referring to one of the two stellar types that give substantial contribution to the broad line formation, namely RGs and SGs in this case) the three quantities $R_{\text {ext }}, \hat{n}_{*}$ and $U$. These are functions of the distance from the central black hole $r$, and we plot two curves for each quantity, corresponding to the two representative values of the expansion velocity parameter $v_{\text {term }}$ previously defined, namely $v_{\text {term }}=0.1 \mathrm{~km} \mathrm{~s}^{-1}$ and $v_{\text {term }}=2.1 \mathrm{~km} \mathrm{~s}^{-1}$, close to the extremes of the velocity range plotted in Fig. 2. For the sake of clarity, we note that the $v_{\text {term }}$ values chosen refer to those attained for envelopes at $r=r_{2}$, that is at the external border of the broad line emitting region. For each BLR physical parameter the curves obtained for intermediate terminal velocity values typically lie in the region between the 0.1 and 2.1 curves. Therefore, each of the two panels of Fig. 3 essentially shows the ranges of values for the BLR parameters for the models corresponding to the present choice of mass loss rates for the stars of the central cluster. It is interesting to notice that for RGs the extension of the BLR contributing region is independent of the terminal velocity value, whereas this is not the case for SGs. This is again due to the fact that for RGs the envelope radius is defined

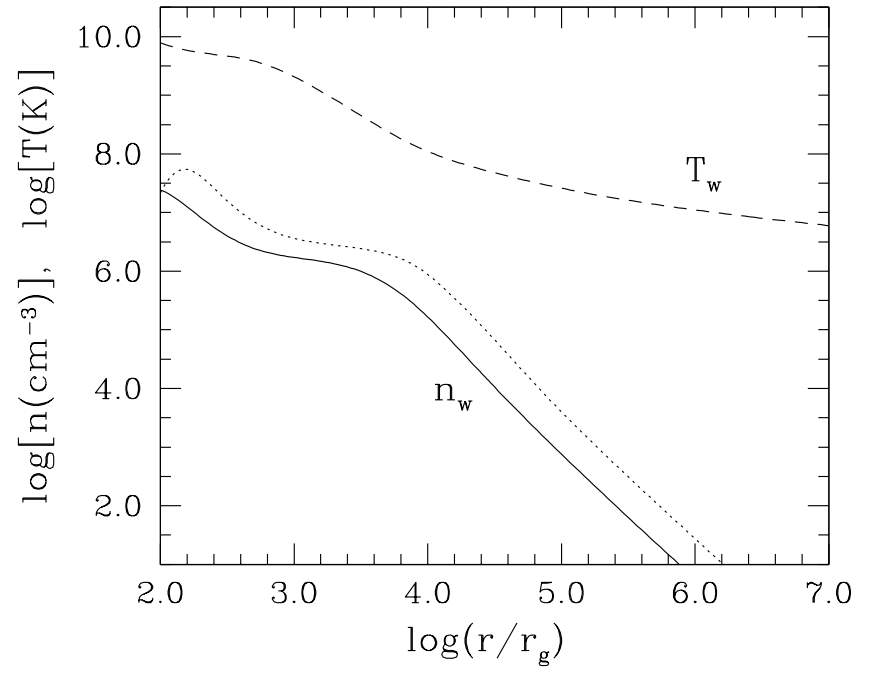

Fig. 4. Temperature (dashed line) and number density (continuous line) profiles for the AGN nuclear wind solution obtained for the case of mass deposition defined by the choice of "standard" mass loss rates for the cluster stars. For comparison, we also plot as the dotted curve the "most extreme" density profile that we have obtained, namely the one resulting from the solution for the nuclear wind with the mass deposition defined by the choice of mass loss rates described as case c) in Sect. 5 and discussed in detail in forthcoming Sect. 5.3.

by the bow-shock mechanism, whereas at low $v_{\text {term }}$ it becomes $\left(R_{\mathrm{ext}}\right)_{\mathrm{SG}}=\left(R_{\mathrm{tidal}}\right)_{\mathrm{SG}}$. Indeed, it is easy to see that, when the bow-shock is the confining physical mechanism for the stellar envelope, and the expansion velocity is subsonic, $n_{*}(r)$ (see Eqs. (6) and (7)) is essentially independent of the stellar wind velocity itself. Moreover, when the envelope gas temperature is chosen to be the same for the three stellar types, $n_{*}(r)$ is independent of the stellar type as well. The same considerations do hold for the average density in the emitting portion of the envelope, $\hat{n}_{*}$ (see Eq. (22)), which is the quantity that we plot in Fig. 3, since in general it turns out that $\hat{n}_{*}=n_{*}$. In fact, our computations have shown that the emitting shell of an envelope is characterized by a size $\Delta R<<R_{\text {ext }}$, so that, performing an analytical integration of Eq. (22) and using a first order approximation in $\Delta R / R_{\text {ext }}(<<1)$, one ends up with $\hat{n}_{*}=n_{*}$. As a result, the limiting conditions on $\hat{n}_{*}$ for line emission (points b) and c) of Sect. 4.2) are met at the same distance from the black hole, $r$, independently of the chosen value for the velocity parameter, since (see Eqs. (5) and (6)) they only depend on the nuclear wind physical properties, namely $n_{\mathrm{w}}(r)$, the nuclear wind density, $T_{\mathrm{w}}(r)$, its temperature profile, and on the velocity defining the ram pressure at the envelope boundary (see Sect. 2). We want to stress here that these same considerations do hold independently of the choice of mass loss rates, therefore they similarly apply to the cases discussed in the next subsections as well. For completeness, in Fig. 4 we show the behaviour of the AGN wind physical quantities $n_{\mathrm{w}}(r)$ and $T_{\mathrm{w}}(r)$, as obtained from the nuclear wind system resolution for this specific choice of the mass deposition. For this density profile the resulting total optical depth to electron scattering for the nuclear wind plasma is $\tau_{\mathrm{T}} \simeq 0.12$. Note that, for comparison, we have also plotted in Fig. 4 the $n_{\mathrm{w}}$ density profile (dotted curve) resulting from the computation of the solution for the nuclear 
wind with a different mass deposition, that is the one that will be extensively discussed in Sect. 5.3, and corresponds to our "most extreme" (i.e., strongest $\dot{M}_{\text {inp }}$ ) choice for mass deposition in the present work; the temperature profile corresponding to this latter case is not shown, since the differences with the dashed curve of Fig. 4 are qualitatively irrelevant.

Going back to the limiting conditions (points b) and c) of Sect. 4.2) for the BLR contributing envelope density $\hat{n}_{*}$, for the present case they define the interval $\left[r_{1}, r_{2}\right]_{\mathrm{RG}} \simeq\left[237 r_{\mathrm{g}}, 1.7 \times\right.$ $\left.10^{4} r_{\mathrm{g}}\right]=\left[3.95 \times 10^{15} \mathrm{~cm}, 2.8 \times 10^{17} \mathrm{~cm}\right]$, thus including any reasonable estimate of the characteristic size $r_{\mathrm{BLR}}$ for our chosen value of $L$.

From Fig. 2, it is apparent that, within the "standard" mass loss rate case defined above, significant covering factors can be recovered only when the terminal velocities of expansion for the stellar envelopes are quite low, as compared with the typical sonic speed for the envelope gas (for $T_{*} \simeq 2 . \times 10^{4} \mathrm{~K}$, we get $c_{\mathrm{s}} \simeq 21 \mathrm{~km} \mathrm{~s}^{-1}$ ). To obtain $C_{\text {tot }} \simeq 0.1, v_{\text {term }}$ should be around $0.027 \mathrm{~km} \mathrm{~s}^{-1}$. Indeed, this is a very low value for the expansion velocity of a "normal" stellar expanding atmosphere. However, we would like to mention that similarly low values have been presented in AN2 (1997) in their own attempt to model the BLR structure as an ensemble of expanding stellar envelopes. In fact, transposing their notation to ours, these authors (AN2) assume $\alpha=-0.5$, a decreasing function of distance from the star surface for the stellar wind velocity, and they choose to analyze models with $v_{0} \simeq 0.1 \mathrm{~km} \mathrm{~s}^{-1}$, where the value of $v_{0}$ is, in their case, the peak value of the stellar wind velocity.

We want to note that the present case of "standard" mass loss rates seems to be not very much relevant to the construction of a physical BLR model for another reason, namely the fact that the calculation of the resulting covering factor for forbidden lines, which are actually not seen in the BLR, gives uncomfortable results. In fact, the global value of $C_{\text {forb }}$ (as defined in Eq. (24)) turns out to reach a significant fraction of the obtained $C_{\text {tot }}$. For example, when $C_{\text {tot }} \simeq 0.1$, we get for the present case $C_{\text {forb }} \simeq 0.037$, which is not negligible. This is related to the characteristics of the nuclear wind solution for the present case, in which not much mass is input in the nuclear wind as a consequence of stellar mass loss, and, therefore, the nuclear wind plasma density $n_{\mathrm{w}}$ is rather low in the "external" (i.e., at $\left.r \gtrsim 10 r_{\mathrm{BLR}}\right)$ region; this allows for the formation of larger expanding envelopes, characterized by density values that are sufficiently low to contribute to the increase of the covering factor for forbidden lines (see the trends shown in Fig. 3 for the envelope physical parameters).

As a final remark, we note that the total number of stars contributing to the BLR, that is essentially the number of RGs in the interval of distances $\left[r_{1}, r_{2}\right]$ for this case, turns out to be $2 \times 10^{5}$. The total number of stars present within the bounds of the BLR for this case is therefore $\sim 2 \times 10^{7}$.

\subsection{Enhanced mass loss rates}

It is by now well known that in a close binary system, intense X-ray illumination from the primary star can have noticeable effects on the secondary star. The best studied example is that of HZ Her (in the system HZ Her/Her X-1), whose illuminated face originates an "X-ray-excited" wind (see e.g. Hameury et al. 1993). The effect is related to the intensity of the X-ray flux at the stellar surface, and from this intensity it is possible to estimate, through an efficiency factor, $\eta$, the fraction of luminosity that gets transformed in kinetic power of the wind. By physical analogy, we suppose that the stars of the AGN central cluster may suffer from a similar effect, since they are strongly illuminated by the hard radiation of the central radiation field of the AGN itself (see, for example, Taylor 1999). We can therefore try an analogous (see Hameury et al. 1993) estimate of the transfer of kinetic power into the stellar wind of stars belonging to our central cluster in a radio-quiet AGN, from the X-ray luminosity, $L_{\mathrm{X}}$, of the radiation field of the AGN central source, as follows:

$\eta L_{\mathrm{X}}\left(\frac{R_{*}}{2 r}\right)^{2} \simeq v_{*}^{2} \dot{M}_{*}$.

The value of $\eta$ computed for Her X-1 is $3 \times 10^{-3}$ and it must be in any case $\eta<0.1$ (Hameury et al. 1993). Taking into account that $L_{\mathrm{X}}<L$, we assume $\eta L_{\mathrm{X}} \simeq 10^{-3} L$. We notice here that, in order to produce BLR models that meet the general observational requirements discussed in Sect. 4.2, the resulting velocities for the expanding stellar envelopes are significantly lower than the sonic speed (by an order of magnitude or even more). Therefore, it seems appropriate to derive an estimate of the "enhanced" mass loss rate for any given type of stars in the cluster with a $v_{*}$ value close to the ones that correspond to models that better fulfill the observational conditions on the BLR. In fact, we choose to use $v_{*} \simeq 3 \mathrm{~km} \mathrm{~s}^{-1}$ to infer an order of magnitude value for the induced mass loss rate of a star in the central cluster of the AGN from relation (27). For $L=10^{44} \mathrm{erg} / \mathrm{s}$, we thus find an estimate for the enhanced mass loss rates

$\left(\dot{M}_{*}\right)_{\mathrm{MS}} \simeq 8 \times 10^{-2}\left(r / r_{\mathrm{g}}\right)^{-2} M_{\odot} / \mathrm{yr}$,

$\left(\dot{M}_{*}\right)_{\mathrm{RG}} \simeq 8\left(r / r_{\mathrm{g}}\right)^{-2} M_{\odot} / \mathrm{yr}$

$\left(\dot{M}_{*}\right)_{\mathrm{SG}} \simeq 8 \times 10^{2}\left(r / r_{\mathrm{g}}\right)^{-2} M_{\odot} / \mathrm{yr}$.

We have computed models using for the three types of stars considered the mass-loss rates given by Eqs. (28) to (30) for $r$ such that those relations give values larger than the "standard" ones defined in Sect. 5.1, and maintaining, on the contrary, those same "standard" values when the equations above would determine smaller values. At $r \sim r_{\mathrm{BLR}}$ the above estimates would give $\left(\dot{M}_{*}\right)_{\mathrm{MS}} \simeq 2.4 \times 10^{-8} M_{\odot} / \mathrm{yr},\left(\dot{M}_{*}\right)_{\mathrm{RG}} \simeq$ $2.4 \times 10^{-6} M_{\odot} / \mathrm{yr}$, and $\left(\dot{M}_{*}\right)_{\mathrm{SG}} \simeq 2.4 \times 10^{-4} M_{\odot} / \mathrm{yr}$. These evaluations point out an interesting consequence of this enhancement mechanism for stellar mass loss rates. In fact, at distances comparable to the characteristic BLR radius, this process does change substantially the "intrinsic" red giant and supergiant mass loss rates, by a factor $\gtrsim 10^{2}$, but, in the specific environment considered here, it has much more influence on the effective mass loss rate of main sequence stars, that turns out to be increased by more than a factor $10^{6}$ at these distances, relevant to the BLR. Therefore, the first important difference with respect to the "standard" mass loss rate case presented in Sect. 5.1 is that the contribution of MS stars to the total covering factor is no longer negligible for any value of the expansion 


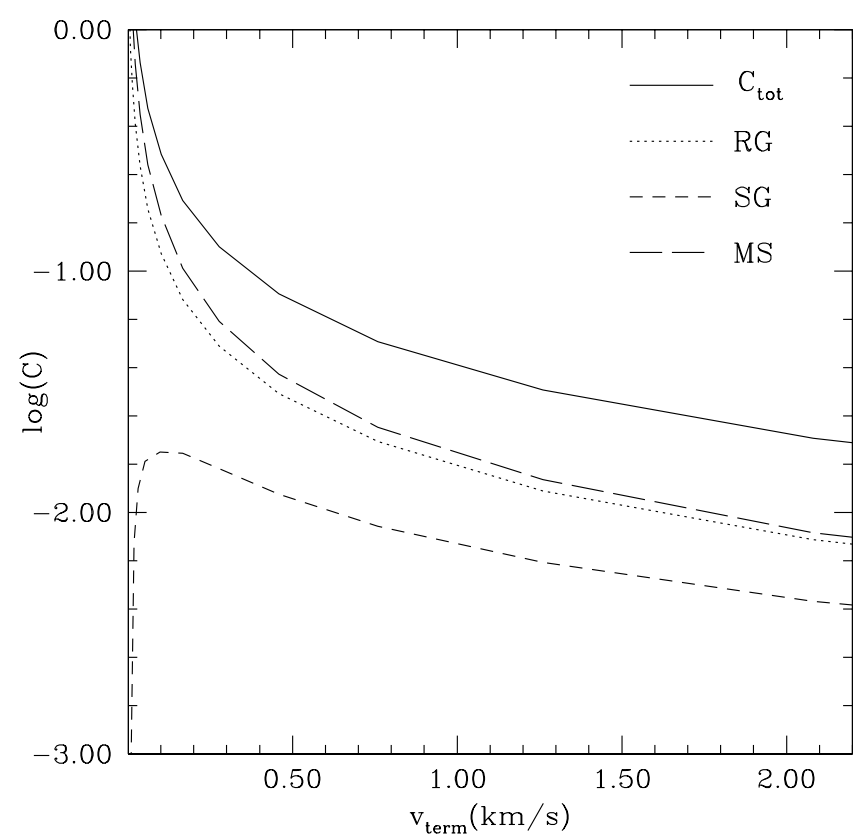

Fig. 5. The total value of the covering factor, $C_{\mathrm{tot}}$, defined as the sum of the contributions due to the envelopes of different stellar types, as well as the distinct contributions to this value from the various stellar types, are shown as functions of the terminal velocity of expansion $v_{\text {term }}$ (in $\mathrm{km} \mathrm{s}^{-1}$ ), characterizing the contributing envelopes. The present figure refers to the case for "enhanced" mass loss rates, and, differently from the case shown in Fig. 2, the contribution due to main sequence stars is quite significant, and actually predominant with respect to RG and SG contributions.

velocity of the emitting envelopes. This is immediately apparent at a glance from Fig. 5, which is the analogous to Fig. 2 discussed in the previous Sect. 5.1 for the case of "standard" mass loss rates. The plot in Fig. 5 shows that the strong increase in mass loss rate for MS stars produces as a consequence a significant contribution to the covering factor. In fact, the extension of the emitting stellar envelopes of MS stars is determined by the bow shock mechanism for basically any expansion velocity and the envelope radius is determined by Eq. (7); therefore, main sequence star envelopes turn out to be much larger than in the case of Sect. 5.1, due to the strong increase in $\left(\dot{M}_{*}\right)_{\mathrm{MS}}$. Again, the number density $n_{\mathrm{w}}(r)$ and temperature $T_{\mathrm{w}}(r)$ of the nuclear wind are computed as the consistent solution of the nuclear wind system of equations with the present mass deposition choice. In this case, the total optical depth to electron scattering for the nuclear wind plasma turns out to be $\tau_{\mathrm{T}} \simeq 0.15$.

It is interesting to note that, due to the $r^{-2}$ decrease in $\left(\dot{M}_{*}\right)_{i}$ moving outwards from the central radiation source, the MS star envelope extension, $R_{\text {ext }}$, shows an extremely slow increase (if not a similarly extremely slow decrease locally) with increasing $r$. This can be seen directly from Fig. 6, similar to Fig. 3 for the "standard" mass loss case discussed in Sect. 5.1 (and we refer to that section for a detailed explanation of the notation and of the choice of the curves plotted); only, in the present case, we show the results referring to MS stars and RG stars in the upper and lower panel respectively, since these two types of stars are the ones that mostly contribute to the present BLR model. As for RG envelopes, their extension is

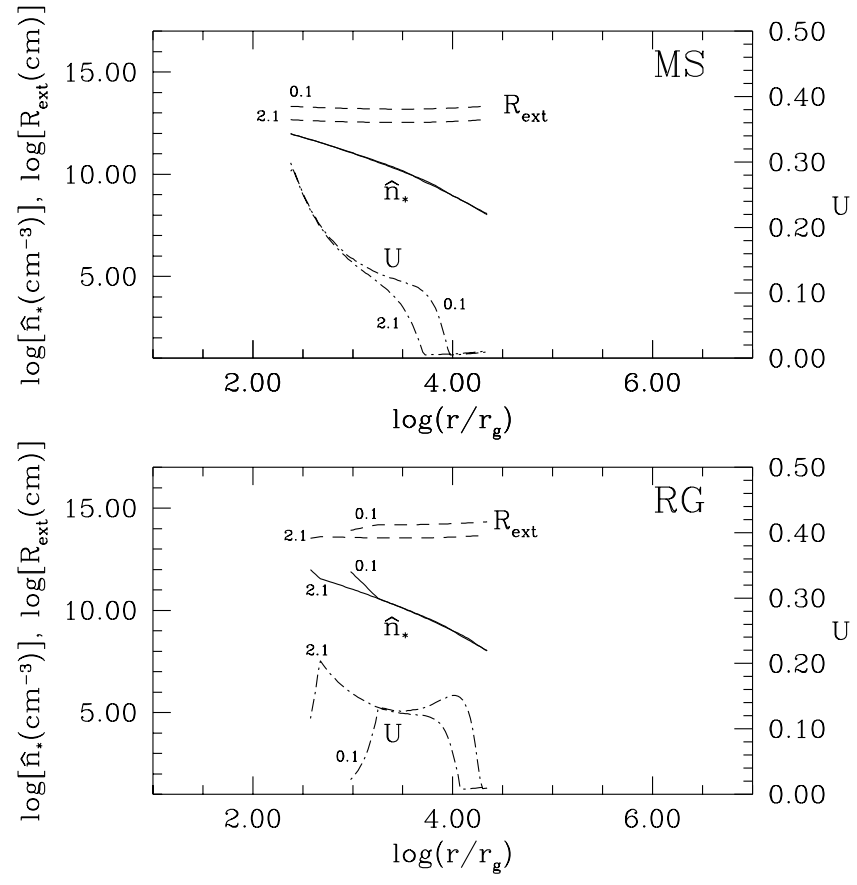

Fig. 6. Physical parameters characterizing stellar envelopes contributing to the BLR as functions of the normalized distance $r / r_{\mathrm{g}}$ from the central black hole; these are the mean density of the envelope, $\hat{n}_{*}(r)$, the estimate of the envelope extension, $R_{\mathrm{ext}}(r)$, and the ionization parameter, $U(r)$. The interval over which the physical quantities are plotted represents the "BLR" interval $\left[r_{1}, r_{2}\right]$ as defined in Sect. 4.2; the labels 0.1 and 2.1 identify the specific value (in $\mathrm{km} \mathrm{s}^{-1}$ ) of the terminal velocity parameter for which they are computed. The upper panel refers to main sequence star envelopes, while the lower one shows the results pertaining to the envelopes of RG stars, in the case of "enhanced" mass loss rates.

mostly bow shock determined, apart from the very inner shells of the BLR, in which the envelope radius is "tidally" defined; this "tidally dominated" portion of the RGs contribution to the BLR slowly extends to larger $r$ distances with decreasing the expansion velocity parameter. We note that this does not influence the behaviour of the curve $C_{\mathrm{RG}}\left(v_{\text {term }}\right)$, since the portion of BLR over which the envelope radius turns out to be "tidal" is in any case not much extended in $r$ and, especially, it is restricted to the inner region of the BLR contributing interval, whereas most of the contribution to the integrated covering factor $C_{\mathrm{RG}}\left(r_{2}\right)$ accumulates at larger values of the distance $r$, in the "bow shock dominated" portion of the interval $\left[r_{1}, r_{2}\right]_{\text {RG }}$. Analogously to the MS envelopes' case, in the "bow shock dominated" region the envelope radius of RG expanding atmospheres is more or less constant with $r$, and this can be seen from the lower panel of Fig. 6. The resulting interval of distances defining the extension of the MS stars contribution to the BLR is $\left[r_{1}, r_{2}\right]_{\mathrm{MS}} \simeq\left[239 r_{\mathrm{g}}, 2.2 \times 10^{4} r_{\mathrm{g}}\right]=\left[4 . \times 10^{15} \mathrm{~cm}\right.$, $\left.3.7 \times 10^{17} \mathrm{~cm}\right]$. RG stars contribute in a region $\left[r_{1}, r_{2}\right]_{\mathrm{RG}}$ whose extension shows a dependence on the velocity parameter $v_{\text {term }}$, as it can be seen from Fig. 6, but it does anyway contain the estimated value of $r_{\mathrm{BLR}}$, independently of $v_{\text {term }}$ itself. As for the mean envelope density $\hat{n}_{*}$, for the conditions in which the envelope radius is $R_{\mathrm{ext}}=R_{\mathrm{bow}}$, we refer to the considerations presented in Sect. 5.1, that still hold. 
From Fig. 5 it is apparent that, at any value of the expansion velocity, the contribution to the total covering factor due to MS stars is dominant, but comparable in order of magnitude with the RG contribution. On the contrary, the contribution of SG envelopes turns out to be much less significant in this case. An analysis of the SG envelope extension as a function of $r$ shows that when the velocity parameter $v_{\text {term }}$ becomes very small $\left(<0.2 \mathrm{~km} \mathrm{~s}^{-1}\right)$, the dominant confinement mechanism for the envelopes becomes generally different from the bow-shock one, similarly to what was discussed for the "standard" mass loss case in Sect. 5.1, thus leading to the change in slope of the curve $C_{\mathrm{SG}}=C_{\mathrm{SG}}\left(v_{\text {term }}\right)$ observed in Fig. 5. As for the definition of the range of values for the velocity parameter $\left(v_{0}\right)_{i}$ for the present case, we report representative values that are analogous to those defined in Sect. 5.1 for the "standard" mass loss rate case, namely $\left(v_{0}\right)_{\mathrm{MS}}\left(v_{\text {term }}=0.1 \mathrm{~km} \mathrm{~s}^{-1}\right)=1.4 \times 10^{-3} \mathrm{~km} \mathrm{~s}^{-1},\left(v_{0}\right)_{\mathrm{RG}}\left(v_{\text {term }}=\right.$ $\left.0.1 \mathrm{~km} \mathrm{~s}^{-1}\right)=1.4 \times 10^{-3} \mathrm{~km} \mathrm{~s}^{-1},\left(v_{0}\right)_{\mathrm{SG}}\left(v_{\text {term }}=0.1 \mathrm{~km} \mathrm{~s}^{-1}\right)=$ $1.4 \times 10^{-3} \mathrm{~km} \mathrm{~s}^{-1}$, and $\left(v_{0}\right)_{\mathrm{MS}}\left(v_{\text {term }}=2.1 \mathrm{~km} \mathrm{~s}^{-1}\right)=8.8 \times$ $10^{-2} \mathrm{~km} \mathrm{~s}^{-1},\left(v_{0}\right)_{\mathrm{RG}}\left(v_{\text {term }}=2.1 \mathrm{~km} \mathrm{~s}^{-1}\right)=8.8 \times 10^{-2} \mathrm{~km} \mathrm{~s}^{-1}$, $\left(v_{0}\right)_{\mathrm{SG}}\left(v_{\text {term }}=2.1 \mathrm{~km} \mathrm{~s}^{-1}\right)=8.8 \times 10^{-2} \mathrm{~km} \mathrm{~s}^{-1}$. Notice that here we have given the values for main sequence stars as well, since they do contribute to the covering factor in this specific case. Also, the values we report are exactly the same independently of the stellar type and this is due to the specific choice of the mass loss rates for the three stellar types, which turn out to give $\left(\dot{M}_{*} / R_{*}^{2}\right)_{\mathrm{MS}}=\left(\dot{M}_{*} / R_{*}^{2}\right)_{\mathrm{RG}}=\left(\dot{M}_{*} / R_{*}^{2}\right)_{\mathrm{SG}}$, thus resulting in the same $\left(R_{\text {ext }} / R_{*}\right)$ value at the BLR external border, defined by the condition $n_{*}=10^{8} \mathrm{~cm}^{-3}$ for any stellar type (see Eq. (3)).

It is easy to see that in the present case, we can build models corresponding to values around 0.1 for the total covering factor for envelope expansion velocities significantly larger than what results in the case of "standard" mass loss rates. In fact, we obtain $C_{\text {tot }} \simeq 0.1$ for $v_{\text {term }} \sim 0.4 \mathrm{~km} \mathrm{~s}^{-1}$, which is more than one order of magnitude larger than the result obtained in the case discussed in Sect. 5.1. However, this velocities still are in a strongly subsonic range of values. Another improvement, with respect to the global results of the first case (discussed in Sect. 5.1), is the fact that for the present choice of stellar mass loss rates, the ratio $C_{\text {forb }} / C_{\text {tot }}$ turns out to be quite smaller, typically ranging between 0.02 and 0.04 for the range of velocity parameter shown in Fig. 5, thus setting the value of $C_{\text {forb }}$ significantly close to "vanishing".

Finally, we want to stress another important difference with respect to the case of the standard mass loss model, namely the fact that in the present scenario we have a much larger number of distinct emitting units constituting the BLR structure, since we can include all main sequence stars of the central cluster characterized by distances $r$ from the central black hole that fall in the BLR contributing interval $\left[r_{1}, r_{2}\right]_{\mathrm{MS}}$. For example, for models giving $C_{\text {tot }} \simeq 0.1$, we get an estimate of the total emitting units in the $\operatorname{BLR}\left(N_{*}\right)_{\text {tot }} \simeq 2.7 \times 10^{7}$.

\section{3. "Intermediate" mass loss rates}

As a third case for stellar mass loss rates, we have chosen another set of $r$-dependent $\left(\dot{M}_{*}\right)_{i}$. In this case, however, we do not specify the physical motivation lying beneath the choice, like in Sect. 5.2, and we only rely on the fact that other mechanisms could be responsible of generating a condition in which mass loss rates of stars in the central cluster of an AGN could be altered (and enhanced) with respect to the "standard" values (see Sect. 6 for a brief discussion). This possibility has been taken into account also in previous analyses of BLR models based on stellar envelopes as individual contributors to the line emission. We refer, for instance, to the work of Scoville \& Norman (1988), in which the authors consider giant star envelopes in a central cluster as emitting units; they suppose that for the giant stars an "induced" mass loss, that turns out to depend on the distance $r$, is generated by some unspecified external heating from the central source. Therefore, our choice in the present section is similar to the one of Scoville \& Norman (1988), since we do not analyse the physical mechanism responsible for affecting the stellar mass loss rates.

We have thus parameterized the mass loss rates that we take into account in the present section as follows:

$$
\begin{aligned}
& \left(\dot{M}_{*}\right)_{\mathrm{MS}}=9 \times 10^{-10}\left(\frac{r}{10^{6} r_{\mathrm{g}}}\right)^{-\beta} M_{\odot} / \mathrm{yr} \\
& \left(\dot{M}_{*}\right)_{\mathrm{RG}}=3 \times 10^{-7}\left(\frac{r}{10^{6} r_{\mathrm{g}}}\right)^{-\beta} M_{\odot} / \mathrm{yr}, \\
& \left(\dot{M}_{*}\right)_{\mathrm{SG}}=3 \times 10^{-6}\left(\frac{r}{10^{6} r_{\mathrm{g}}}\right)^{-\beta} M_{\odot} / \mathrm{yr}
\end{aligned}
$$

where we have chosen $\beta=0.3$. For any type of star, the equations above define the mass loss rate that we use for the models we discuss in this section for $r$ up to the distance at which the value of the mass loss rate itself (which is a decreasing function of $r$ ) reaches the "standard" value mentioned at the beginning of Sect. 5.1, whereas for larger distances we discard Eqs. (31)-(33) and we assume that the mass loss rate is constant and given by the "standard" values of Sect. 5.1. As for the nuclear wind number density and temperature profiles $\left(n_{\mathrm{w}}(r)\right.$ and $T_{\mathrm{w}}(r)$ ) derived from the nuclear wind solution referring to the present case for mass deposition, we remind that $n_{\mathrm{w}}(r)$ is shown in Fig. 4 as the dotted curve. The nuclear wind temperature profile shows no significant differences with respect to the one shown in Fig. 4, from a qualitative point of view. The resulting total optical depth to electron scattering for the nuclear wind plasma is here $\tau_{\mathrm{T}} \simeq 0.4$.

We want to note that, with respect to the case presented in the previous section, for red giants the present choice of mass loss rate function has significantly larger values over most of the region from which we expect to have BLR contributions. A similar condition holds for MS stars as well, but the effect is weaker than for RGs. As for SG stars, the situation is less well defined, but $\left(\dot{M}_{*}\right)_{\mathrm{SG}}(r)$ is effectively larger than what we get in Sect. 5.2 over the outer portion of the region contributing to the BLR emission for SGs. As a consequence of this, the resulting contributions of the different types of stars to the total covering factor, that we again show as functions of the expansion velocity parameter $v_{\text {term }}$ in Fig. 7, turn out to have a different relative importance with respect to the "enhanced" mass loss rate case of Sect. 5.2. In fact, in the present case RG stars are the 


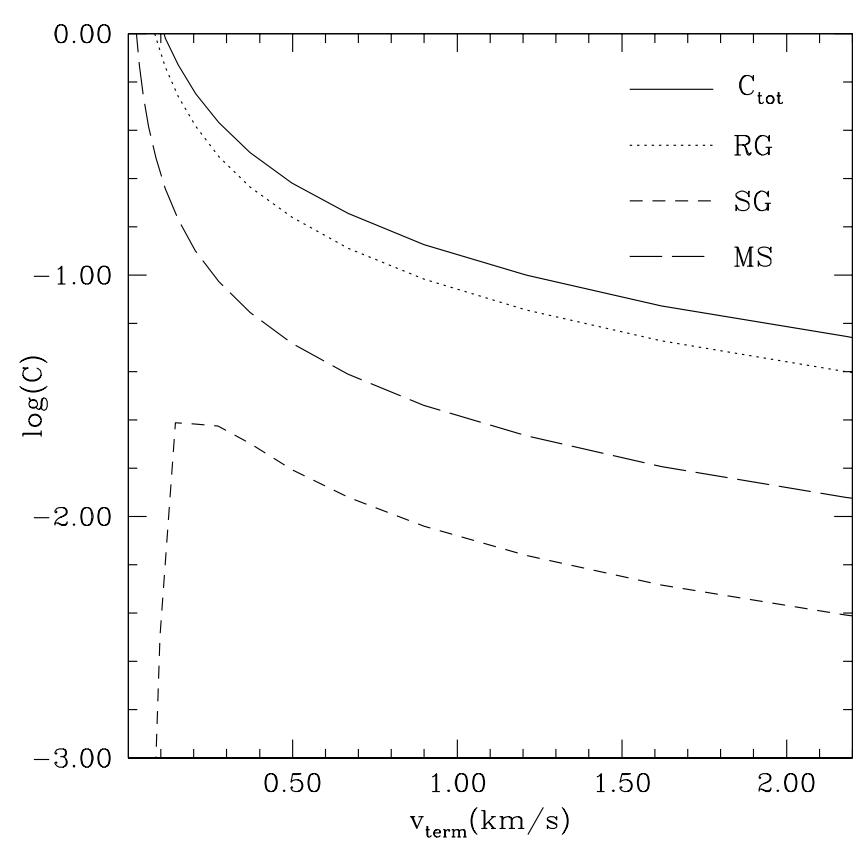

Fig. 7. The total value of the covering factor, $C_{\mathrm{tot}}$, defined as the sum of the contributions due to the envelopes of different stellar types, as well as the distinct contributions to this value from the various stellar types, are shown as functions of the terminal velocity of expansion $v_{\text {term }}$ (in $\mathrm{km} \mathrm{s}^{-1}$ ), characterizing the contributing envelopes. In the present "intermediate" case, both RGs and MSs give substantial contribution to the definition of the total covering factor.

dominant contributors to the total covering factor, but the contribution of main sequence stars is significant ( $\sim 22 \%$ of $\left.C_{\text {tot }}\right)$ at any value of the velocity parameter $v_{\text {term }}$. On the contrary, the contribution due to much less numerous SG stellar component does not increase as much, and we get a $C_{\mathrm{SG}}$ estimate which is $\leq 7 \%$ of the total value of the covering factor for any $v_{\text {term }}$.

A closer inspection of the BLR models we have used to build the curves in Fig. 7 shows again that the bow-shock mechanism for confinement of the stellar expanding envelopes is the one that effectively determines the extension of the envelopes under any condition for MS stars. For RG envelopes this is still true, except for the very low velocity end of the interval shown, in which case other confinement mechanisms dominate in the inner portion ( $r \lesssim 500 r_{\mathrm{g}}$ ) of the BLR contributing region, $\left[r_{1}, r_{2}\right]_{\mathrm{RG}}$. On the other hand, SG envelopes are confined basically by bow-shock mechanism for $v_{\text {term }}>0.3 \mathrm{~km} \mathrm{~s}^{-1}$, whereas for lower values of the velocity parameter other confinement mechanisms become more efficient in defining the envelope extension, just like the case analysed in Sect. 5.2.

Regarding the definition of the range of values for the velocity parameter $\left(v_{0}\right)_{i}$ for the present case, again we report representative values analogous to those defined in Sects. 5.1 and 5.2 for the previous mass loss rate cases, namely $\left(v_{0}\right)_{\mathrm{MS}}\left(v_{\mathrm{term}}=\right.$ $\left.0.1 \mathrm{~km} \mathrm{~s}^{-1}\right)=5.8 \times 10^{-4} \mathrm{~km} \mathrm{~s}^{-1},\left(v_{0}\right)_{\mathrm{RG}}\left(v_{\text {term }}=0.1 \mathrm{~km} \mathrm{~s}^{-1}\right)=$ $3.7 \times 10^{-4} \mathrm{~km} \mathrm{~s}^{-1},\left(v_{0}\right)_{\mathrm{SG}}\left(v_{\text {term }}=0.1 \mathrm{~km} \mathrm{~s}^{-1}\right)=8.8 \times$ $10^{-4} \mathrm{~km} \mathrm{~s}^{-1}$, and $\left(v_{0}\right)_{\mathrm{MS}}\left(v_{\text {term }}=2.1 \mathrm{~km} \mathrm{~s}^{-1}\right)=3.4 \times$ $10^{-2} \mathrm{~km} \mathrm{~s}^{-1},\left(v_{0}\right)_{\mathrm{RG}}\left(v_{\text {term }}=2.1 \mathrm{~km} \mathrm{~s}^{-1}\right)=2.1 \times 10^{-2} \mathrm{~km} \mathrm{~s}^{-1}$, $\left(v_{0}\right)_{\mathrm{SG}}\left(v_{\text {term }}=2.1 \mathrm{~km} \mathrm{~s}^{-1}\right)=5.1 \times 10^{-2} \mathrm{~km} \mathrm{~s}^{-1}$. We have given the values for main sequence stars as well, since they give a substantial contribution to the covering factor also in this case.

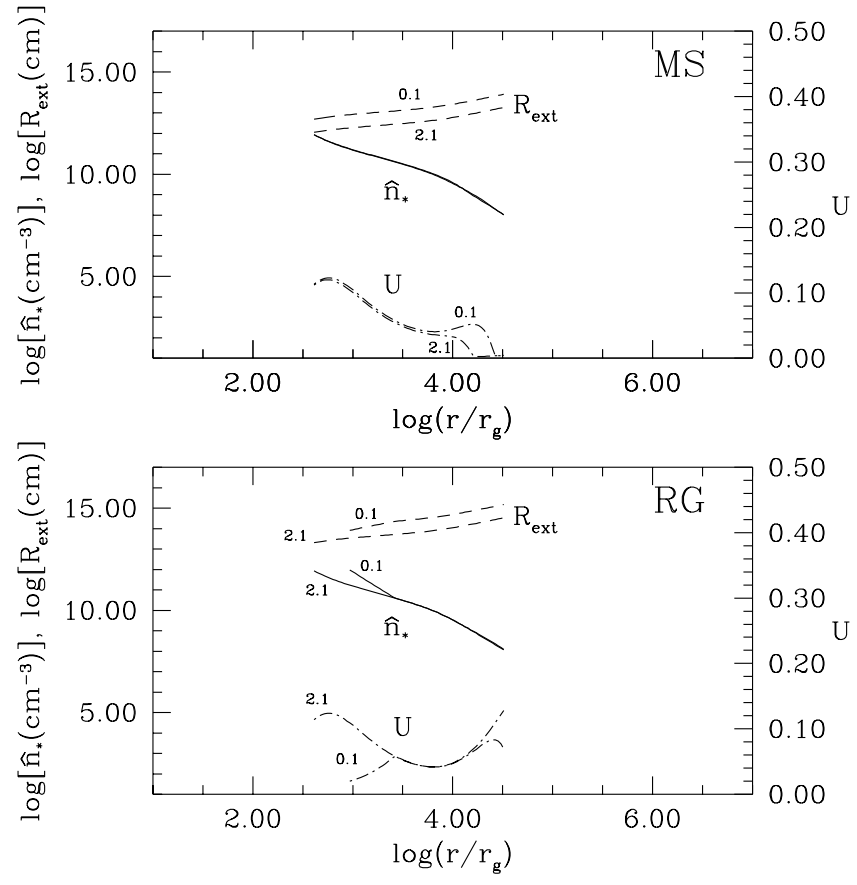

Fig. 8. Physical parameters characterizing stellar envelopes contributing to the BLR as functions of the normalized distance $r / r_{\mathrm{g}}$ from the central black hole; these are the mean density of the envelope, $\hat{n}_{*}(r)$, the estimate of the envelope extension, $R_{\text {ext }}(r)$, and the ionization parameter, $U(r)$. The interval over which the physical quantities are plotted represents the "BLR" interval $\left[r_{1}, r_{2}\right]$ as defined in Sect. 4.2; the labels 0.1 and 2.1 identify the specific value (in $\mathrm{km} \mathrm{s}^{-1}$ ) of the terminal velocity parameter for which they are computed. We show the results for both MSs (upper panel) and RGs (lower panel), since they both significantly contribute to the total covering factor in the present case of "intermediate" mass loss rates.

Similarly to Figs. 3 and 6 for the previous two cases, Fig. 8 shows the profiles of the relevant physical parameters for stellar envelopes contributing to the BLR as functions of the distance $r$. We show the results pertaining to the same two representative values of the velocity parameter $v_{\text {term }}$, namely $0.1 \mathrm{~km} \mathrm{~s}^{-1}$ and $2.1 \mathrm{~km} \mathrm{~s}^{-1}$. The extension of the BLR contributing interval for MS envelopes is $\left[r_{1}, r_{2}\right]_{\mathrm{MS}}=\left[410 r_{\mathrm{g}}, 3.2 \times\right.$ $\left.10^{4} r_{\mathrm{g}}\right] \simeq\left[6.8 \times 10^{15} \mathrm{~cm}, 5.3 \times 10^{17} \mathrm{~cm}\right]$, independently of the velocity parameter, again because of the fact that the extension of the envelopes is always defined by the bow-shock mechanism. For RG envelopes, the BLR contributing interval has the same upper limit $r_{2}$ as that for MS contributors, whereas the inner boundary $r_{1}$ shows a dependence on the velocity parameter (see previous paragraph).

As it is easy to see from Fig. 7, the present choice of mass loss rates for the central cluster stars turns out to be the most favourable one from the point of view of the value of the covering factor that can be recovered with our models. In particular, the interval of observationally inferred values for a Seyfert 1 BLR, defined in Sect. 4.2 to be ranging from 0.05 up to around 0.25 , can be obtained with stellar envelopes characterized by expansion velocities in the range $0.5-3.0 \mathrm{~km} \mathrm{~s}^{-1}$, which corresponds to subsonic, but physically sensible values for an expanding stellar atmosphere. As for the values of $C_{\text {forb }}$, they turn out to be sufficiently small to be considered "vanishing", 
similar to what happens in the case for "enhanced" mass loss rates of Sect. 5.2, since they at most reach $6 \%$ of $C_{\text {tot }}$.

As a final consideration for the intermediate mass loss scenario we estimate total number of emitting units. Indeed, similar to the case for "enhanced" mass loss rates, we can include MS star envelopes in the total number of individual contributors to the model BLR, since their contribution to the total covering factor is also substantial in this case. This allows an estimate of $\left(N_{*}\right)_{\text {tot }} \sim 3.2 \times 10^{7}$ stars; this number is even a little higher that the one estimated for the case of "enhanced" mass loss rates; this is due to the fact that in the present case the external boundary of the BLR contributing interval of distances is larger than that of the case discussed in Sect. 5.2, thus allowing to include a larger number of stars in the computation.

\section{General discussion}

The results presented in the previous section show that our model is capable of reproducing the observational requirements listed in Sect. 4.2 (points 1-4). In fact, from Figs. 3, 6 and 8 it is evident that the ionization parameter $U$ is in the expected range of values, while $r_{\mathrm{BRL}}$ is contained in the intervals $\left[r_{1}, r_{2}\right]$ where the conditions a)-d) of Sect. 4.2 are satisfied. As far as the covering factor is concerned, it is evident that its value strongly depends on the choice adopted for the stellar mass loss rates (compare Figs. 2, 5 and 7). In the case of enhanced and intermediate mass loss rates, values in the range $0.05-0.25$ can be recovered by choosing suitable values of the stellar envelope expansion velocities, while for standard mass loss rates this is possible only for very low velocity values. Hence, our values of $C_{\text {tot }}$ tend to exclude stellar envelopes with standard mass loss rates as plausible emitting units for a BLR model. On the other hand, regarding the covering factor for "broad" forbidden line emission, we recall that the star density distribution we have adopted has been chosen so that in all the presented cases $C_{\text {forb }}$ is minimized (see Sect. 5); in fact, it turns out to be negligible in both the cases presented in Sects. 5.2 and 5.3. We recall here that in the "standard" mass loss rate case, $C_{\text {forb }}$ is not negligible and we consider this one of the reasons why that particular model is not viable for building up a realistic BLR model.

While the analysis of this paper has produced strong constraints to most of the free model parameters, for some, namely the stellar distribution, the mass loss rates and the expansion velocity, the model cannot operate a definite choice. Any further test of the model and selection of these parameters requires more information coming from the comparison between the observed line profiles and relative intensities, and those predicted by the model. As we have already mentioned, we have planned to compute the detailed line profiles in a forthcoming paper, having now obtained a deeper insight in the role that different parameters play in the model. However, at this stage we would like to compare our model with the results of some other authors (Goad \& Koratkar 1998; Kaspi \& Netzer 1999; Korista \& Goad 2000) who made attempts to infer from line profiles and intensities some "average" physical conditions in BLR condensations.

In the last years much work has been devoted to infer the BLR physical configuration starting from the observed BLR properties. Since the BLR emitting gas has been shown to be stratified in density (see Peterson 1993 and Netzer \& Peterson 1997 for reviews) the idea was to derive both the dependence of the characteristic number density of the gas in the "cloudlike" emitting clumps $\left(\hat{n}_{*}(r)\right.$ in our notation) and that of the "cloud-like" structures' distribution $\left(\rho_{*}(r)\right.$ in our notation) on the radial distance $r$ from the central black hole. This analysis has been applied by the authors mentioned above to the case of the well studied and representative Seyfert 1 NGC 5548.

Combining a photoionization code with an optimization routine, Goad \& Koratkar (1998) have found that line ratios and variability time scales can be reproduced assuming $\hat{n}_{*}(r) \propto r^{-2}$. Kaspi \& Netzer (1999), through the "direct" method of guessing cloud properties and distribution and calculating the resulting emission lines, have also found that a density gradient is necessary but their best fit is $\hat{n}_{*}(r) \propto r^{-\mathrm{s}}$ with $1 \leq s<1.5$. In our results $\hat{n}_{*}(r)$ slope is variable in the interval $\left[r_{1}, r_{2}\right]$ where star envelopes contribute to build up the covering factor. For $\hat{n}_{*}(r)$ profiles shown in Figs. 3, 6 and 8 a piece-wise power law approximation gives logarithmic slopes from 1.5 up to 2 . These values cannot be used to exclude or to prefer one of the assumed mass loss profiles, since they do not contradict any of the two previously quoted analyses.

Recently Korista \& Goad (2000) have tested the "locally optimally emitting clouds" (LOC) model, proposed by Baldwin et al. (1995), by comparing the predicted spectrum with that of NGC 5548. One of the results they attain is that the power law index of the quantity they define as the radial cloud covering fraction must be in the range -1.6 to -0.5 . In our notation this implies that the product $R_{\mathrm{ext}}^{2}(r) \rho_{*}(r)$ must be a function of $r$ which is representable by a power law whose exponent should be in the range $[-1.6,-0.5]$ mentioned above. Since the star distribution function $\rho_{*}(r)$ that we have adopted has a logarithmic slope ranging from -0.7 around $r / r_{\mathrm{g}}=10^{3}$ down to -2 around $r / r_{\mathrm{g}}=10^{4}$, this comparison favours $R_{\mathrm{ext}}(r)$ functions flatter than $r^{0.75}$. This fact again implies that "enhanced" and "intermediate" mass loss rates are preferable, since in these cases the functional form of $R_{\text {ext }}(r)$ does indeed fulfill the requirement above (in fact, it turns out that it is $\propto r^{0.66}$ or flatter both for MS and RG stars).

Both Korista \& Goad (2000) and Kaspi \& Netzer (1999) derive an estimate for the extension of the BLR. For the first ones the maximum BLR extension can be up to 200 light days, while for the second ones it can be up to 100 light days. In our model, the values for the derived external BLR radius, $r_{2}$, for the case of "intermediate" mass loss (see Sect. 5.3) are larger than those derived for the case of "enhanced" mass loss rates presented in Sect. 5.2; however, they are in any case consistent with the estimate obtained by Korista \& Goad (2000). Again, this comparison is encouraging for our model, but not definite enough to allow for a selection of most appropriate mass loss rate values. In any case, the general comparison of our model with the global inferred physical conditions for the BLR discussed above favours the two configurations analyzed in Sects. 5.2 and 5.3, characterized by distance dependent stellar mass loss rates and especially by a substantial contribution to BLR emission coming from MS star envelopes, whose mass loss rate is strongly enhanced with respect to their "standard" value. 
Besides the X-ray illumination mechanism that we have discussed and examined in Sect. 5.2, other mechanisms have been analyzed in literature, that support the physical plausibility of this type of induced and $r$-dependent stellar mass loss rates. Here we just want to mention a couple of these works. For example, MacDonald et al. (1991) performed an analysis of the effects of neutrino and high energy particle flux from the central source on the stars of the central cluster and their winds; their main result is that these stellar winds can be affected in the sense of a mass loss enhancement. Also, Baldwin et al. (1996) have computed the radiative acceleration on the photospheric layers of stars at BLR distances for a number of high-luminosity AGNs; comparing this acceleration with the star surface gravity, they conclude that all the stars, including main sequence ones, can indeed be affected, thus producing an enhanced mass loss. We recall that for the case of $r$ dependent and enhanced mass loss rates that we have discussed in Sect. 5.3, we have not explicitly defined the physical mechanism inducing the enhancement, similarly to what is done in Scoville \& Norman (1988) (see Sect. 5.3), but we just supposed one of such mechanisms to be at work.

Going back to what we have pointed out above, the two cases discussed in Sects. 5.2 and 5.3 fall in the same "category" of models, characterized by strongly enhanced mass loss rates and a significant contribution to the BLR emission coming from MS stars. This is interesting from two different, but connected, points of view. First, within this picture the large number of individual emitting units does not imply too large collision rates between the stars. In fact, the star collision rate (Eq. (18)) relative to the star distribution, $\rho_{*}(r)$, for which we have computed the models whose results are shown in Sect. 5 (the one labeled with "1" in Fig. 1) turns out to be $\tau \simeq 1$ collisions/yr. The total star collision rate is an estimate of the total number of stellar collisions that take place in the whole cluster in a year; however, to understand to what degree the cluster structure is affected by stellar collisions another quantity must be introduced, that is the time that it takes for a star to be destroyed by collisions with other stars belonging to the cluster. Following Begelman \& Sikora (1992), we introduce here the destruction time due to mutual collisions for a star in the cluster as

$t_{\mathrm{coll}}(r) \simeq \frac{10}{\rho_{*}(r) f_{i} \pi\left(R_{*}\right)_{i}^{2} V(r)}$,

where the number 10 at numerator is the number of collisions that a star has to undergo to be finally disrupted; the value used above is estimated from the fraction of stellar mass lost per collision that Begelman \& Sikora (1992) evaluate as $\sim 0.1$. In our model the destruction time per star turns out to be the same for red giant and main sequence stars (since $\left(R_{*}\right)_{\mathrm{RG}}=10\left(R_{*}\right)_{\mathrm{MS}}$ and $f_{\mathrm{RG}}=0.01 f_{\mathrm{MS}}$ ) and it varies with the distance $r$ from the central black hole. To derive an estimate of its value, we have computed it in the region of interest for this work, that is at a distance which is around a half of the estimated external radius $\left(r_{2}\right)$ of the BLR model, i.e. $\sim 10^{4} r_{\mathrm{g}}$, obtaining $t_{\text {coll }}\left(x=10^{4}\right) \simeq 8 \times 10^{8}$ yr. A necessary condition for our picture to be consistent is that the MS star evolution time is shorter than the star destruction time. Taking into account the value of $t_{\text {coll }}$ derived above, this condition is verified for stars with masses larger than $2.6 M_{\odot}$ which, in a star cluster of $3 \times 10^{7}$ stars, assuming the Salpeter initial mass function, constitute $\sim 3 \%$ of the total star number. This percentage of stars can account for the assumed quantity of evolved stars in our model. Obviously, going closer to the cluster center, $t_{\text {coll }}$ decreases and this condition can no longer be fulfilled. However, there are a few arguments that suggest that the destruction time for collisions computed above should be considered as a sort of lower limit evaluation. In fact, as Scoville \& Norman (1988) argue, ordered stellar motions may result in a lower collision rate. In addition, as discussed by these same authors, stars orbiting on elongated elliptical orbits would spend only a short part of their life in the inner region of the cluster and this would result in a larger survival probability as well. Our present discussion is centered on survival conditions for the central stellar cluster as we have chosen to model it. Its relation to the AGN lifetime (possibly $<10^{8} \mathrm{yr}$ ) and to the time at which the nuclear activity switches on with respect to the evolutionary stage of the cluster itself would indeed deserve further insight. However, in the present context, it is probably just the case to mention the possible relevance of this issue and we leave its analysis to different work. Back to the original point, even accounting for these last considerations, the discussion above shows that the very large values of the star number density characterizing the stellar distribution $\rho_{*}(r)$ that we have chosen for our models (the one labelled with 1 in Fig. 1) can be regarded as very close to the upper limit for the maintainance of the cluster integrity. Indeed, higher stellar densities would result in a cluster evolution much faster than what is required when the cluster star envelopes are believed to represent the structural components of an AGN Broad Line Region. As a matter of fact, this is indeed the case when the only stellar populations forming expanding envelopes suitable for Broad Line emission (i.e., contributing to the BLR) are those of RGs and SGs. In fact, both RG and SG populations amount to only a small fraction of the total stellar number in the cluster (i.e., $\sim 1 \%$, see Sect. 4.1 ); as a consequence, requiring a number density of RG and SG envelopes, as contributing emitting units, sufficiently large to justify the observationally inferred covering factors implies a total number density of stars around 100 times larger. This would lead to a destruction time (as estimated through Eq. (34)) unacceptably short (see Begelman \& Sikora 1992).

In addition, a second point of interest regarding the significant contribution of MS star envelopes to the BLR emission, is that, owing to the large number of emitting structures, our model can be easily set in accordance with the results of the studies of bright AGN spectra, performed with cross-correlation techniques, such as those of Arav et al. (1997, 1998), that claim to determine a lower limit on the number of individual emitting structures of the BLR, for models based on discrete emitting units composing the region. In particular, for Mk 335, these same authors derive a lower limit for the number of reprocessing cloud-like structures, which is expected to be around $3 \times 10^{6}$.

To compare our model to the results inferred by Arav et al. $(1997,1998)$ for Mk 335, we have to take into account configurations with a more powerful central AGN nucleus. Therefore, 
we have analyzed models for $L=10^{45} \mathrm{erg} / \mathrm{s}$, that is the luminosity of this source. To model an AGN with this luminosity, we have chosen the same central black hole mass as that shown in Table 1 of Paper I, namely $M_{\mathrm{BH}}=1.12 \times 10^{8} M_{\odot}$; also, for simplicity, we suppose that the central star cluster has just the same profile $\rho_{*}(r)$ as the one adopted for the case of luminosity $L=10^{44} \mathrm{erg} / \mathrm{s}$ (see the curve labeled with "1" in Fig. 1). With these hypotheses, we can test the resulting total number of stars within a distance corresponding to the estimated external boundary of the BLR model, comparing it with Arav et al. $(1997,1998)$ lower limit evaluation of the number of individually emitting units in the source mentioned. Our result turns out to be $\left(N_{* \text { tot }}\right)_{45} \simeq 3.9 \times 10^{7}$ stars, where we have computed this number using the estimate we obtain for $\left(r_{2}\right)_{\mathrm{MS}} \sim 2.3 \times 10^{4} r_{\mathrm{g}}$, since the total number of stars is essentially determined by the number of MS stars in the cluster for scenarii of the type described in Sects. 5.2 and 5.3. Our evaluation of $\left(N_{* \text { tot }}\right)_{45}$ turns out to be well above the lower limit found by Arav et al. (1997, 1998), and this is another encouraging outcome. As for other properties of broad line emitting stellar envelopes in this same case of "high" luminosity ( $L=10^{45} \mathrm{erg} / \mathrm{s}$ ) AGN, these turn out to show behaviours that are similar to those we have discussed in Sects. 5.2 and 5.3 for the case for $L=10^{44} \mathrm{erg} / \mathrm{s}$. We note in passing that the bow shock mechanism for envelope confinement is in general still the most efficient, although in this higher luminosity case Comptonization confinement (see Sect. 2, Eq. (12)) can be dominant in the inner $\left(r \lesssim 500 r_{\mathrm{g}}\right)$ portion of the model broad line emitting zone (that is, closer to the central luminosity source, where the radiation flux is stronger).

As a final remark, we note that the fact that the model outcome in this scenario can be set in accordance with the very large number of discrete broad line emitters in the region required by the analysis performed by Arav et al. $(1997,1998)$ could be of relevance with respect to the BLR structure problem, because it would show that discrete emitting units models of the BLR are not necessarily ruled out by the analysis of Arav et al. (1998).

\section{Conclusions}

In this paper we have analyzed the scenario in which BLR emission originates in expanding atmospheres of the stars of a dense central cluster embedded in the hot, tenuous plasma outflowing from the AGN. Immersing the central star cluster in its specific AGN environment and investigating the interaction of the stellar wind with the AGN outflow, we consider the shock fronts generated by the interaction of the stellar wind and AGN outflow as another confining mechanism for the stellar envelopes. In our model, the nuclear wind structure depends on the features of the dense stellar cluster around the central black hole (including the mass loss rates of the stellar components of the cluster itself). At the same time, the physical conditions of the stellar envelopes emitting the broad lines depend on the nuclear wind properties. Thus, looking for the stellar envelope physical conditions that are appropriate to reproduce the general characteristics of the BLR as they are deduced from observations, we have solved the problem consistently.
We have identified those parameters that significantly influence the resulting BLR model properties: these turn out to be $v_{0}$, a stellar wind velocity parameter, the set $\left\{\dot{M}_{\mathrm{MS}}, \dot{M}_{\mathrm{RG}}, \dot{M}_{\mathrm{SG}}\right\}$ of stellar mass loss rates, and $\rho_{*}(r)$, the star number density distribution. Indeed, we have explored different possibilities for building a BLR composed of mass losing stellar envelopes, determining, in the very specific and extreme AGN central region environment, the conditions under which different star types contribute to the observed line emission. In summary, we have selected two main choices for the BLR structure, namely 1) a scenario in which stars are taken as characterized by their "standard" (i.e., as known from solar neighbourhood studies) mass loss rate, examined in Sect. 5.1, and 2) another one in which stars are characterized by an enhanced mass loss rate. Both cases, the "enhanced" mass loss rates and the "intermediate" mass loss rates, examined in Sects. 5.2 and 5.3, belong to this second picture. In the first scenario, red giant and supergiant envelopes are the foremost contributors to BLR, while in the second, main sequence stars turn out to be an important constituent of the BLR.

For both scenarios, we have built configurations that meet the basic physical conditions for stellar extended atmospheres to be able to contribute to BLR emission. As discussed in Sect. 6, the observational requirements are much better fulfilled by models built in the second scenario, allowing for large mass loss rates, with respect to those that we can construct starting from the assumption of standard stellar mass loss rates. In addition, if we compare the two scenarios with the further conditions inferred from a global analysis of BLR observations and with the deductions of Arav et al. $(1997,1998)$ on the total number of individually emitting structures in the BLR, again we find a differentiation in behaviour and outcomes. The scenario in which main sequence stars are also endowed with winds capable of producing stellar envelopes with a nonnegligible mass loss rate is therefore preferable. This point shows the importance of the choice of the mass loss rate in our model.

Besides the above general considerations, in our model the most stringent quantity turns out to be $C_{\text {tot }}$. In particular, covering factors that match the range derived from observations are not easily obtained. To recover values of $C_{\text {tot }}$ as large as those observationally inferred we need to suitably choose the star number density $\rho_{*}(r)$ and/or the ratio $\dot{M} / v_{0}$. The star number density distribution of the cluster orbiting around the central black hole turns out to be crucial in our model. In fact, the observed BLR features can not be recovered for any given $\rho_{*}(r)$ : while the required global amount of stars in the cluster is not large, the star number density in the central region (i.e. around $10^{16} \mathrm{~cm}$ ) must be rather high. Also, high mass loss rates and low outflow velocities are preferable.

Even with these limitations, the model nevertheless constitutes a consistent structure for a BLR embedded in an AGN wind-type plasma outflow, with individual emitting units characterized by the appropriate values of physical quantities for broad line emission. Of course, a more detailed comparison of the line shape and intensity produced by our model would be necessary to strengthen the validity of this approach. 
As a first step, however, we can assert that the results we obtain are encouraging, because this analysis has determined strong constraints on the free parameters in the model. Such constraints will be useful in the derivation of the characteristic line profiles that can be predicted by our model.

Acknowledgements. We thank Nick Scoville and Leslie Hunt for helpful discussions; it is also a pleasure to acknowledge Leslie Hunt's careful reading of the paper. This work was partly supported by the Italian Ministry for University and Research (MURST) under grant Cofin00-02-36.

\section{References}

Alexander, T., \& Netzer, H. 1994, MNRAS, 270, 781 (AN1)

Alexander, T., \& Netzer, H. 1997, MNRAS, 284, 967 (AN2)

Alexander, T. 1997, MNRAS, 285, 891

Allen, C. W. 1985, Astrophysical Quantities (The Athlone Press, London)

Arav, N., Barlow, T. A., Laor, A., \& Blandford, R. D. 1997, MNRAS, 288,1015

Arav, N., Barlow, T. A., Laor, A., Sargent, W. L. W., \& Blandford, R. D. 1998, MNRAS, 298, 990

Baldwin, J., Ferland, G., Korista, K., \& Verner, D. 1995, ApJ, 455, L119

Baldwin, J., Ferland, G. J., Korista, K. T., et al. 1996, ApJ, 461, 664

Baldwin, J. A. 1997, in Emission Lines in Active Galaxies: New Methods and Techniques, ed. B. M. Peterson, F. Z. Cheng, \& A. S. Wilson (San Francisco: ASP), ASP Conf. Ser., 113, 80

Begelman, M. C., \& Sikora, M. 1992, in Testing the AGN Paradigm, Proc. AIP Conf. 254, ed. S. S. Holt, S. G. Neff, \& C. M. Urry (AIP, New York), 568

Crenshaw, D. M., Kraemer, S. B., Boggess, A., et al. 1999, ApJ, 516, 750

David, L. P., Durisen, R. H., \& Cohn, H. N. 1987a, ApJ, 313, 556

David, L. P., Durisen, R. H., \& Cohn, H. N. 1987b, ApJ, 316, 505

Elvis, M., Wilkes, B. J., McDowell, J. C., et al. 1994, ApJS, 95, 1

Goad, M. R., \& Koratkar, A. 1998, ApJ, 495, 718

Hameury, J. M., King, A. R., Lasota, J. P., \& Raison, F. 1993, A\&A, 277,81
Kaspi, S. 1997, in Emission Lines in Active Galaxies: New Methods and Techniques, ed. B. M. Peterson, F.-Z. Cheng, \& A. S. Wilson (San Francisco: ASP), ASP Conf. Ser., 113, 159

Kaspi, S., \& Netzer, H. 1999, ApJ, 524, 71

Kaspi, S., Smith, P. S., Netzer, H., et al. 2000, ApJ, 533, 631

Kaspi, S., Brandt, W. N., Netzer, H., et al. 2001, ApJ, 554, 216

Kazanas, D. 1989, ApJ, 347, 74

Korista, K. 1999, in Quasars and Cosmology, ASP Conf. Ser., ed. G. Ferland, \& J. Baldwin (San Francisco: ASP) [astro-ph/9812043]

Korista, K., \& Goad, M. R. 2000, ApJ, 536, 284

Krolik, J. H., McKee, C. F., \& Tarter, C. B. 1981, ApJ, 249, 422

Krolik, J. H. 1999, Active Galactic Nuclei (Princeton University Press)

Krolik, J. H., \& Kriss, G. A. 2001, ApJ, 561, 684

Krolik, J. H. 2002 [astro-ph/0204418]

Lamers, H. J. G. L. M., \& Cassinelli, J. P. 1999, Introduction to Stellar Winds (Cambridge University Press, Cambridge)

MacDonald, J., Stanev, T., \& Biermann, P. L. 1991, ApJ, 378, 30

Murphy, B. W., Cohn, H. N., \& Durisen, R. H. 1991, ApJ, 370, 60

Netzer, H., 1990, in Active Galactic Nuclei, ed. T. J.-L. Courvoisier, \& M. Mayor (Springer Verlag, Berlin), 57

Netzer, H., \& Peterson, B. M. 1997, in Astronomical Time Series, Proc. of the Wise Observatory 25th Ann. Symp., ed. D. Maoz, A. Sternberg, \& E. Leibovitz (Kluwer Academic Publishers, Dordrecht) [astro-ph/9706039]

Perry, J. J., \& Dyson, J. E. 1985, MNRAS, 213, 665

Peterson, B. M. 1993, PASP, 105, 247

Peterson, B. M. 1997, Introduction to Active Galactic Nuclei (Cambridge University Press, Cambridge)

Pietrini, P., \& Torricelli-Ciamponi, G. 2000, A\&A, 363, 455 (Paper I)

Rees, M. J., Netzer, H., \& Ferland, G. J. 1989, ApJ, 347, 640

Scoville, N., \& Norman, C. 1988, ApJ, 332, 163

Taylor, J. A. 1999, Ph.D. Thesis Line Emission from Stellar Winds in AGNs (University of Maryland, College Park)

van Buren, D., Mac Low, M.-M., Wood, D. O. S., \& Churchwell, E. 1990, ApJ, 353, 570

Wandel, A., Peterson, B. M., \& Malkan, M. A. 1999, ApJ, 526, 579

Weymann, R. J., Morris, S. L., Gray, M. E., et al. 1997, ApJ, 483, 717 